OPEN ACCESS

Edited by:

Holmes Finch,

Ball State University, United States

Reviewed by:

Luís Faísca,

University of Algarve, Portugal

Luz Anyela Morales Quintero,

Meritorious Autonomous University

of Puebla, Mexico

*Correspondence:

Flávia de Lima Osório

flaliosorio@gmail.com

tThese authors have contributed equally to this work and share first authorship

Specialty section: This article was submitted to

Quantitative Psychology and Measurement, a section of the journal

Frontiers in Psychology

Received: 22 September 2021

Accepted: 26 October 2021

Published: 24 November 2021

Citation:

Lima FFd and Osório FdL (2021)

Empathy: Assessment Instruments and Psychometric Quality A Systematic Literature Review With

a Meta-Analysis of the Past Ten Years. Front. Psychol. 12:781346. doi: 10.3389/fpsyg.2021.781346

\section{Empathy: Assessment Instruments and Psychometric Quality - A Systematic Literature Review With a Meta-Analysis of the Past Ten Years}

\author{
Felipe Fernandes de Lima ${ }^{1 \dagger}$ and Flávia de Lima Osório ${ }^{1,2 * \dagger}$ \\ 'Ribeirão Preto Medical School, University of São Paulo, São Paulo, Brazil, ${ }^{2}$ National Institute for Science and Technology \\ (INCT-TM, CNPq), Brasília, Brazil
}

Objective: To verify the psychometric qualities and adequacy of the instruments available in the literature from 2009 to 2019 to assess empathy in the general population.

Methods: The following databases were searched: PubMed, Psyclnfo, Web of Science, Scielo, and LILACS using the keywords "empathy" AND "valid*" OR "reliability" OR "psychometr*." A qualitative synthesis was performed with the findings, and metaanalytic measures were used for reliability and convergent validity.

Results: Fifty studies were assessed, which comprised 23 assessment instruments. Of these, 13 proposed new instruments, 18 investigated the psychometric properties of instruments previously developed, and 19 reported cross-cultural adaptations. The Empathy Quotient, Interpersonal Reactivity Index, and Questionnaire of Cognitive and Affective Empathy were the instruments most frequently addressed. They presented good meta-analytic indicators of internal consistency [reliability, generalization metaanalyses (Cronbach's alpha): 0.61 to 0.86 ], but weak evidence of validity [weak structural validity; low to moderate convergent validity $(0.27$ to 0.45$)]$. Few studies analyzed standardization, prediction, or responsiveness for the new and old instruments. The new instruments proposed few innovations, and their psychometric properties did not improve. In general, cross-cultural studies reported adequate adaptation processes and equivalent psychometric indicators, though there was a lack of studies addressing cultural invariance.

Conclusion: Despite the diversity of instruments assessing empathy and the many associated psychometric studies, there remain limitations, especially in terms of validity. Thus far, we cannot yet nominate a gold-standard instrument.

Keywords: empathy, psychometrics, validity, reliability, instruments

\section{INTRODUCTION}

There is growing consensus among researchers concerning empathy being a multidimensional phenomenon in recent years, which necessarily includes cognitive and emotional components (Davis, 2018). Reniers et al. (2011), for instance, consider that empathy comprises both an understanding of other peoples' experiences (cognitive empathy) and an ability to feel their 
emotional experiences (affective empathy) indirectly. BaronCohen $(2003,2004)$ considers that empathy is the ability to identify what other people are thinking and feeling (cognitive empathy) and to respond to these mental states with appropriate emotions (affective empathy), enabling individuals to understand other peoples' intentions, anticipate their behavior and experience the emotions that arise from this contact with people. Hence, empathy enables effective interaction in the social world.

According to Kim and Lee (2010), empathy can be assessed by self-report instruments/scales and observed through most psychological constructs. Moya-Albiol et al. (2010) stress that new strategies have been recently proposed to approach this construct from an ecological perspective, such as computational tasks with emotional stimuli.

The literature presents instruments to assess an individual's ability to provide empathic responses in general and instruments designed to assess empathy in specific contexts, such as ethnocultural empathy (Rasoal et al., 2011), empathy in the face of anger and pain (Vitaglione and Barnett, 2003; Giummarra et al., 2015), empathy among physicians (Alcorta-Garza et al., 2016), health workers, and patients (Scarpellini et al., 2014) and empathy involved in the relationship between teachers and students (Warren, 2015), among others.

Previous studies, such as systematic reviews, have assessed the psychometric quality of instruments intended to assess empathy in some of these specific contexts. Hemmerdinger et al. (2007) assessed the reliability and validity of scales used to assess empathy in medicine, analyzing 36 different instruments. The Medical Condition Regard Scale, Jefferson Scale of Physician Empathy, Consultation and Relational Empathy, and Four Habits Coding Scheme, which were developed for this specific population, stood out together with Davis Interpersonal Reactivity Index (IRI), Empathy Test, Empathy Construct Rating Scale, and Balanced Emotional Empathy Scale, which assess empathic ability in general, as they presented satisfactory psychometric qualities. However, the authors highlighted that instruments focusing on selecting candidates for the medical program lacked sufficient predictive validity evidence. Nonetheless, they concluded that there were measures with sufficient evidence to investigate the role of empathy in the medical and clinical care fields.

Later, Yu and Kirk (2009) attempted to verify the existence of a gold-standard instrument to assess empathy within the nursing field. They identified 12 instruments, $33.3 \%$ of these were originally developed with nursing workers and students (e.g., Empathy Construct Rating Scale and Layton Empathy Test), 33.3\% addressed health workers and patients (e.g., Barrett-Lennard Relationship Inventory and Carkhuff Indices of Discrimination and Communication), and 33.3\% were developed to assess empathic response in general (e.g., Emotional Empathy Tendency Scale and IRI). The results show that most instruments presented not very robust validity or reliability indicators, while less than $15 \%$ of the instruments verified the responsiveness item. The authors concluded that no instrument could be recommended as the gold standard but noted that the Empathy Construct Rating Scale gathered the most robust evidence.
Hong and Han (2020) recently conducted a systematic review to identify scales assessing empathy among health workers in general. Eleven studies were included in the review, among which the Consultation and Relational Empathy, Jefferson Scale of Physician Empathy, and Therapist Empathy Scale (TES). These scales stood out in terms of psychometric quality; however, like previous reviews, the conclusion was that there were no instruments with desirable psychometric qualities to be considered the gold standard. Additionally, none of the measures were specifically developed for professionals working with the elderly, which indicates an important gap in the field.

To our knowledge, no systematic reviews focus on instruments that measure empathic ability in the general population. Hence, this review aimed to describe the psychometric quality and adequacy of instruments available in the literature from 2009 to 2019 to assess empathy in the general population.

\section{MATERIALS AND METHODS}

This study complied with the recommendations proposed by the Preferred Reporting Items for Systematic Review and MetaAnalyses - PRISMA (Moher et al., 2009) and the methodological guidelines established by the BRASIL. Ministério da Saúde et al. (2014). The following databases were searched: PubMed, PsycINFO, Web of Science, Scielo, and LILACS together with the keywords empathy, valid*, reliability, and psychometr*. Inclusion criteria were studies: (a) addressing 18-year-old or older individuals in the general population of both sexes; (b) published between 2009 and 2019, regardless of the language; and (c) with the objective to develop and/or assess the psychometric quality of instruments measuring empathic response in the general population. Figure 1 presents the exclusion criteria and the entire process used to select the studies.

Two mental health workers experienced in psychological and psychometric assessments (FFL, FLO) independently decided on the studies' eligibility; divergences were resolved by consensus. A standard form was developed to extract the following variables: (a) year of publication; (b) study's objective; (c) sample characteristics (i.e., country of origin, sample size, sex, age, and education); (d) instrument's characteristics (objective, number of items, application format, and scoring); and (e) psychometric indicators concerning validity and reliability.

The framework proposed by Andresen (2000) was used to assess the psychometric quality of the papers included in this review. It rates different criteria on a nominal scale ranging from A (strong adequacy) to C (weak or no adequacy), namely: Norms, Standard values; Measurement model; Item/instrument bias; Respondent burden; Administrative burden; Reliability; Validity; Responsiveness; Alternate/accessible forms, and Culture/language adaptations. This review's authors independently assessed the studies' psychometric quality and resolved divergences by achieving a consensus. The definitions of psychometric qualities and assessment criteria are presented in Supplementary Material 1.

A qualitative synthesis of the results was performed for each instrument. Additionally, for those instruments with more than 


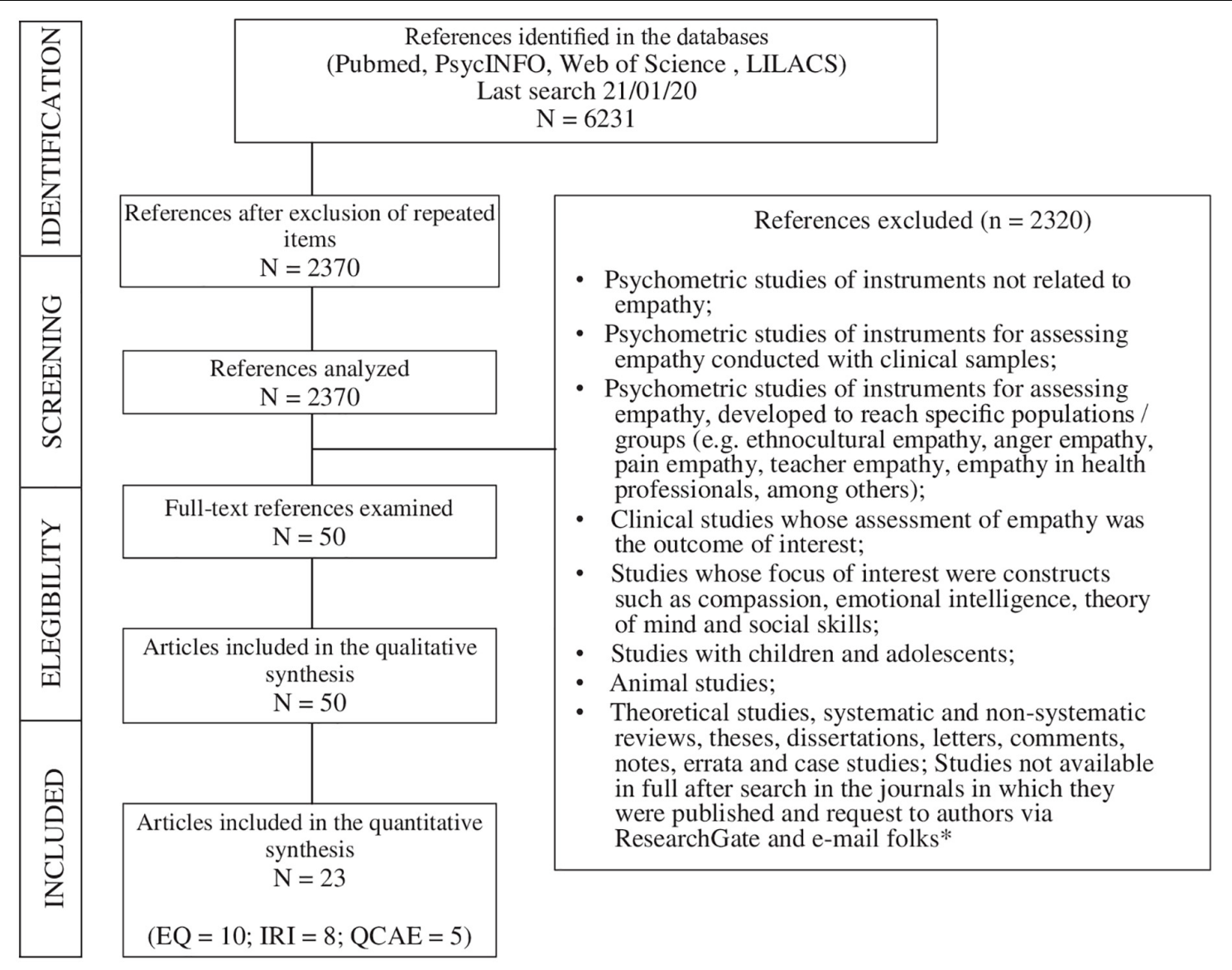

FIGURE 1 | Flowchart describing to the inclusion and exclusion criteria processes based on PRISMA protocol (*Bora and Baysan, 2009; Innamorati et al., 2015).

two studies, meta-analytic measures of reliability and convergent validity were produced using the Jamovi software. We conduct reliability generalization meta-analysis of Cronbach's alpha (for the total scale and/or subscales) (Pentapati et al., 2020), and intraclass correlation coefficients (ICC) were grouped for the computation of test-retest meta-analytic measures (Macchiavelli et al., 2020). To group data concerning convergent validity with empathy measures and correlate constructs, we used Pearson/Spearman correlation coefficient ( $r$ ) as the effect size measure (Duckworth and Kern, 2011). In the case of multiple indicators, the largest indicator in absolute values was chosen. Untransformed estimates and inverse variance weighting were used (Hedges and Olkin, 1985). An average coefficient and a 95\% confidence interval $(95 \% \mathrm{CI})$ were calculated for each metaanalysis. Heterogeneity of the measures between studies was verified using Q-statistic and I2 index. The funnel plot was used to assess the publication bias (Egger et al., 1997).

\section{RESULTS}

Fifty studies were selected, and 23 different instruments were identified. The instruments most frequently addressed were the Empathy Quotient (EQ; $n=11)$, IRI $(n=10)$, and Questionnaire of Cognitive and Affective Empathy (QCAE; $n=5$ ). Only one or two studies assessed each of the remaining instruments. A total of $60.9 \%$ of the instruments were developed in the period included in this review, from 2009 to 2019. The remaining studies were developed before 2009, and the studies assessed new aspects of their psychometric qualities and/or cross-cultural adaptations. Table 1 presents an overview of each instrument.

Table 1 shows that most instruments are self-report scales $(n=21)$, rated on a Likert scale $(70 \%$ included five-point scales), with the number of items ranging from one to 80 (median $=23$ ). Three instruments present alternative versions with fewer items [EQ, IRI, and Empathy Assessment Index (EAI)]. In most cases, data were collected face-to-face $(n=12)$, while the Active-Empathic Listening Scale (AELS) (Drollinger et al., 2006) was the only instrument with an other-report version. Two instruments consisted of computational tasks with the presentation of photorealistic stimuli: the Multifaceted Empathy Test (MET) (Dziobek et al., 2008) and the Pictorial Empathy Test (PET) (Lindeman et al., 2018). Data concerning the samples used by the different studies are presented in Table 2 .

As shown in Table 2, the smallest sample was composed of 50 participants, and the largest sample had 5.724 participants $($ mean $=1036.6 \pm 1577.5)$. Regarding age, most studies 
TABLE 1 | Overview of the instruments analyzed to assess general empathic capacity in the general population (ranked from most studied to least studied).

\begin{tabular}{|c|c|c|c|c|c|c|c|}
\hline Instrument & Author/year & Objective & $\begin{array}{c}\text { Type of } \\
\text { application }\end{array}$ & $\begin{array}{c}\begin{array}{c}\text { Type of } \\
\text { instrument }\end{array}\end{array}$ & No. items & Rating scale & $\begin{array}{l}\text { No. of psychometric } \\
\text { studies (last } 10 \text { years) }\end{array}$ \\
\hline $\begin{array}{l}\text { Empathy Quotient } \\
\text { (EQ-60) }\end{array}$ & $\begin{array}{l}\text { Baron-Cohen and } \\
\text { Wheelwright (2004) }\end{array}$ & $\begin{array}{l}\text { To measure the cognitive and } \\
\text { affective aspects of empathy }\end{array}$ & Presential/Online & SRF & $\begin{array}{l}60 \\
40 \\
22 \\
15\end{array}$ & $\begin{array}{l}\text { Likert - } 4 \text { points } \\
1 \text { ("strongly disagree") to } 4 \text { ("strongly } \\
\text { agree") }\end{array}$ & 11 \\
\hline $\begin{array}{l}\text { Interpersonal Reactivity } \\
\text { Index (IRI) }\end{array}$ & Davis (1983) & $\begin{array}{l}\text { To evaluate empathy through } 4 \\
\text { factors: fantasy, empathic concern, } \\
\text { perspective taking and personal } \\
\text { distress. }\end{array}$ & Presential/Online & SRF & $\begin{array}{l}28 \\
26 \\
16\end{array}$ & $\begin{array}{l}\text { Likert - } 5 \text { points } \\
0 \text { ("doesn't describe me at all") to } 4 \\
\text { (“describes } \\
\text { me very well") }\end{array}$ & 10 \\
\hline $\begin{array}{l}\text { Questionnaire of } \\
\text { Cognitive and Affective } \\
\text { Empathy (QCAE) })^{\star}\end{array}$ & Reniers et al. (2011) & $\begin{array}{l}\text { To evaluate empathy through } 5 \\
\text { factors: perspective taking, online } \\
\text { simulation, emotion contagion, } \\
\text { peripherical responsivity and proximal } \\
\text { responsivity. }\end{array}$ & Presential/Online & SRF & 31 & $\begin{array}{l}\text { Likert - } 4 \text { points } \\
1 \text { ("strongly disagree") to } 4 \text { ("strongly } \\
\text { agree") }\end{array}$ & 5 \\
\hline $\begin{array}{l}\text { The Active-Empathic } \\
\text { Listening Scale (AELS) }\end{array}$ & $\begin{array}{l}\text { Drollinger et al. } \\
\qquad(2006)\end{array}$ & $\begin{array}{l}\text { Computerized task to evaluate } \\
\text { empathy through } 3 \text { factors: sensing, } \\
\text { processing, responding. }\end{array}$ & Presential & $\mathrm{SRF} / \mathrm{HRF}$ & 11 & $\begin{array}{l}\text { Likert }-7 \text { points } \\
1 \text { ("never or almost never true") to } 7 \\
\text { ("always or almost always true") }\end{array}$ & 2 \\
\hline $\begin{array}{l}\text { The Toronto Empathy } \\
\text { Questionnaire (TEQ)* }\end{array}$ & Spreng et al. (2009) & $\begin{array}{l}\text { To assess cognitive and affective } \\
\text { aspects of empathy }\end{array}$ & Presential & SRF & 16 & $\begin{array}{l}\text { Likert }-5 \text { points } \\
1 \text { ("never") to } 5 \text { ("always") }\end{array}$ & 2 \\
\hline $\begin{array}{l}\text { Empathy Assessment } \\
\text { Index }\left(\text { EAl) }{ }^{\star}\right.\end{array}$ & Gerdes et al. (2011) & $\begin{array}{l}\text { To evaluate empathy through } 5 \\
\text { factors: } \\
\text { affective response, perspective } \\
\text { taking, emotion regulation, } \\
\text { self-awareness and empathic } \\
\text { attitudes. }\end{array}$ & Online & SRF & $54 / 50$ & $\begin{array}{l}\text { Likert - } 5 \text { points } \\
1 \text { (“never") to } 5 \text { (“always") }\end{array}$ & 2 \\
\hline $\begin{array}{l}\text { Affective and Cognitive } \\
\text { Measure of Empathy } \\
(\text { (ACME) })^{\star}\end{array}$ & $\begin{array}{l}\text { Vachon and Lynam } \\
\qquad(2016)\end{array}$ & $\begin{array}{l}\text { To evaluate empathy through } 3 \\
\text { factors: cognitive empathy, affective } \\
\text { resonance and affective dissonance }\end{array}$ & Presential/Online & SRF & 36 & $\begin{array}{l}\text { Likert - } 5 \text { points } \\
1 \text { ("strongly disagree") to } 5 \text { ("strongly } \\
\text { agree") }\end{array}$ & 2 \\
\hline $\begin{array}{l}\text { The Mexican Empathy } \\
\text { Scale (MxES) }\end{array}$ & $\begin{array}{l}\text { Díaz-Loving et al. } \\
\qquad(1986)\end{array}$ & $\begin{array}{l}\text { To evaluate empathy through } 4 \\
\text { factors: empathic compassion, } \\
\text { cognitive empathy, Indifference and } \\
\text { disruption }\end{array}$ & Presential & SRF & 32 & $\begin{array}{l}\text { Likert - } 5 \text { points } \\
1 \text { ("does not describe me well") to } \\
\text { ("describes me very well") }\end{array}$ & 1 \\
\hline $\begin{array}{l}\text { Multidimensional } \\
\text { Emotional Empathy } \\
\text { Scale (MDEES) }\end{array}$ & $\begin{array}{l}\text { Caruso and Mayer } \\
\text { (1998) }\end{array}$ & $\begin{array}{l}\text { To evaluate empathy through } 6 \\
\text { factors: suffering, positive sharing, } \\
\text { responsive crying, emotional } \\
\text { attention, feel for other and emotional } \\
\text { contagion }\end{array}$ & Presential & SRF & 30 & $\begin{array}{l}\text { Likert - } 5 \text { points } \\
1 \text { (“Strongly disagree") to } 5 \text { ("Strongly } \\
\text { agree") }\end{array}$ & 1 \\
\hline $\begin{array}{l}\text { Basic Empathy Scale } \\
\text { (BES) }\end{array}$ & $\begin{array}{l}\text { Jolliffe and } \\
\text { Farrington (2006) }\end{array}$ & $\begin{array}{l}\text { To assess cognitive and affective } \\
\text { aspects of empathy }\end{array}$ & Presential & SRF & 20 & $\begin{array}{l}\text { Likert - } 5 \text { points } \\
1 \text { ("strongly disagree") to } 5 \text { ("strongly } \\
\text { agree") }\end{array}$ & 1 \\
\hline Empathy Inventory (EI) & Falcone et al. (2008) & $\begin{array}{l}\text { To evaluate empathy through } 4 \\
\text { factors: } \\
\text { perspective taking; Interpersonal } \\
\text { flexibility; altruism and affective } \\
\text { sensitivity }\end{array}$ & Presential & SRF & 40 & $\begin{array}{l}\text { Likert - } 5 \text { points } \\
1 \text { ("never") to } 5 \text { ("always") }\end{array}$ & 1 \\
\hline $\begin{array}{l}\text { Multifaceted Empathy } \\
\text { Test (MET) }\end{array}$ & Dziobek et al. (2008) & $\begin{array}{l}\text { Computerized task to measure the } \\
\text { cognitive and affective aspects of } \\
\text { empathy }\end{array}$ & Presential & SRF & 80 & $\begin{array}{l}\text { Cognitive test: to select the emotional } \\
\text { state of the depicted person out of a } \\
\text { set of four possible answers. } \\
\text { Affective test: Likert - } 9 \text { points } \\
1 \text { ("not at all") to } 9 \text { ("very strongly") }\end{array}$ & 1 \\
\hline
\end{tabular}


TABLE 1 | (Continued)

\begin{tabular}{|c|c|c|c|c|c|c|c|}
\hline Instrument & Author/year & Objective & $\begin{array}{c}\text { Type of } \\
\text { application }\end{array}$ & $\begin{array}{c}\text { Type of } \\
\text { instrument }\end{array}$ & No. items & Rating scale & $\begin{array}{l}\text { No. of psychometric } \\
\text { studies (last } 10 \text { years) }\end{array}$ \\
\hline $\begin{array}{l}\text { The Vicarious } \\
\text { Experience Scale (VES) }\end{array}$ & $\begin{array}{l}\text { López-Pérez et al. } \\
\text { (2008) }\end{array}$ & $\begin{array}{l}\text { To evaluate empathy through } 2 \\
\text { factors: } \\
\text { sympathy and vicarious distress }\end{array}$ & Presential & SRF & 15 & $\begin{array}{l}\text { Likert - } 5 \text { points } \\
1 \text { (“not at all") to } 5 \text { (“extremely") }\end{array}$ & 1 \\
\hline $\begin{array}{l}\text { Interpersonal and } \\
\text { Social Empathy Index } \\
\text { (ISEI) })^{\star}\end{array}$ & Segal et al. (2013) & $\begin{array}{l}\text { To assess cognitive and affective } \\
\text { aspects of empathy }\end{array}$ & Online & SRF & 15 & $\begin{array}{l}\text { Likert - } 6 \text { points } \\
1 \text { ("never") to } 6 \text { ("always") }\end{array}$ & 1 \\
\hline $\begin{array}{l}\text { Measure of Empathy } \\
\text { and Sympathy (MES)* }\end{array}$ & Vossen et al. (2015) & To measure empathy and sympathy & Online & SRF & 12 & $\begin{array}{l}\text { Likert - } 5 \text { points } \\
1 \text { ("never") to } 5 \text { ("always") }\end{array}$ & 1 \\
\hline $\begin{array}{l}\text { Positive Empathy Scale } \\
\text { (PES) }^{*}\end{array}$ & Morelli et al. (2015) & To evaluate positive empathy & Presential & SRF & 7 & $\begin{array}{l}\text { Likert - } 5 \text { points } \\
1 \text { (“does not describe me at all") to } \\
5 \text { ("describes me very well") }\end{array}$ & 1 \\
\hline $\begin{array}{l}\text { Empathic Behavior } \\
\text { Scale }(E C E)^{\star}\end{array}$ & Auné et al. (2017) & $\begin{array}{l}\text { To assess the demonstration of the } \\
\text { other's understanding, the ability to } \\
\text { put oneself in the other's place, the } \\
\text { ability to support the other, make } \\
\text { positive reinforcements through } \\
\text { speech, share experiences, take } \\
\text { action to reduce conflict and } \\
\text { emotional support in painful } \\
\text { moments. }\end{array}$ & Presential & SRF & 8 & $\begin{array}{l}\text { Likert - } 5 \text { points } \\
1 \text { ("never") to } 5 \text { ("always") }\end{array}$ & 1 \\
\hline $\begin{array}{l}\text { Empathy Components } \\
\text { Questionnaire (ECQ) }\end{array}$ & $\begin{array}{l}\text { Batchelder et al. } \\
\text { (2017) }\end{array}$ & $\begin{array}{l}\text { To evaluate empathy through } 5 \\
\text { factors: cognitive ability, cognitive } \\
\text { drive, affective ability, affective drive } \\
\text { and affective reactivity. }\end{array}$ & Online & SRF & 30 & $\begin{array}{l}\text { Likert - } 4 \text { points } \\
1 \text { ("strongly disagree") to } 4 \\
\text { ("strongly agree") }\end{array}$ & 1 \\
\hline $\begin{array}{l}\text { Empathy Gradient } \\
\text { Questionnaire }(E G Q)^{*}\end{array}$ & Hollar (2017) & $\begin{array}{l}\text { To evaluate empathy through } 5 \\
\text { factors: } \\
\text { family empathy, friend empathy, } \\
\text { peer/colleague empathy, distant } \\
\text { other empathy, and species } \\
\text { empathy. }\end{array}$ & Presential & SRF & 23 & $\begin{array}{l}\text { Likert - } 5 \text { points } \\
1 \text { ("I very much } \\
\text { disagree") to } 5 \text { ("I very much agree") }\end{array}$ & 1 \\
\hline $\begin{array}{l}\text { Cognitive, Affective, } \\
\text { and Somatic Empathy } \\
\text { Scales (CASES) }\end{array}$ & Raine et al. (2018) & $\begin{array}{l}\text { To evaluate empathy through } 3 \\
\text { factors: cognitive, affective, and } \\
\text { somatic empathy }\end{array}$ & Presential & SRF & 30 & $\begin{array}{l}\text { Likert - } 4 \text { points } \\
0 \text { ("rarely") to } 3 \text { ("often") }\end{array}$ & 1 \\
\hline $\begin{array}{l}\text { Online Empathy } \\
\text { Questionnaire (QoE)* }\end{array}$ & Miguel et al. (2018) & $\begin{array}{l}\text { To evaluate empathy through } 3 \\
\text { factors: } \\
\text { responsiveness, respect and } \\
\text { availability. }\end{array}$ & Online & SRF & 23 & $\begin{array}{l}\text { Likert - } 5 \text { points } \\
1 \text { ("never") to } 5 \text { ("always") }\end{array}$ & 1 \\
\hline $\begin{array}{l}\text { Pictorial Empathy Test } \\
(\mathrm{PET})^{\star}\end{array}$ & $\begin{array}{l}\text { Lindeman et al. } \\
\qquad(2018)\end{array}$ & $\begin{array}{l}\text { Computerized task to evaluate } \\
\text { affective empathic }\end{array}$ & Online & SRF & 7 & $\begin{array}{l}\text { Likert - } 5 \text { points } \\
1 \text { (“not at all") to } 5 \text { ("always") }\end{array}$ & 1 \\
\hline $\begin{array}{l}\text { Single Item Trait } \\
\text { Empathy Scale (SITES)* }\end{array}$ & $\begin{array}{l}\text { Konrath et al. } \\
\text { (2018) }\end{array}$ & $\begin{array}{l}\text { To measure empathy through a } \\
\text { single item }\end{array}$ & Online & SRF & 1 & $\begin{array}{l}\text { Likert - } 5 \text { points } \\
1 \text { ("not very true of me") to } 5 \text { ("very } \\
\text { true of me") }\end{array}$ & 1 \\
\hline
\end{tabular}


TABLE 2 | Characterization of the samples used by the different studies ( $N=50$ - ranked from most studied to least studied).

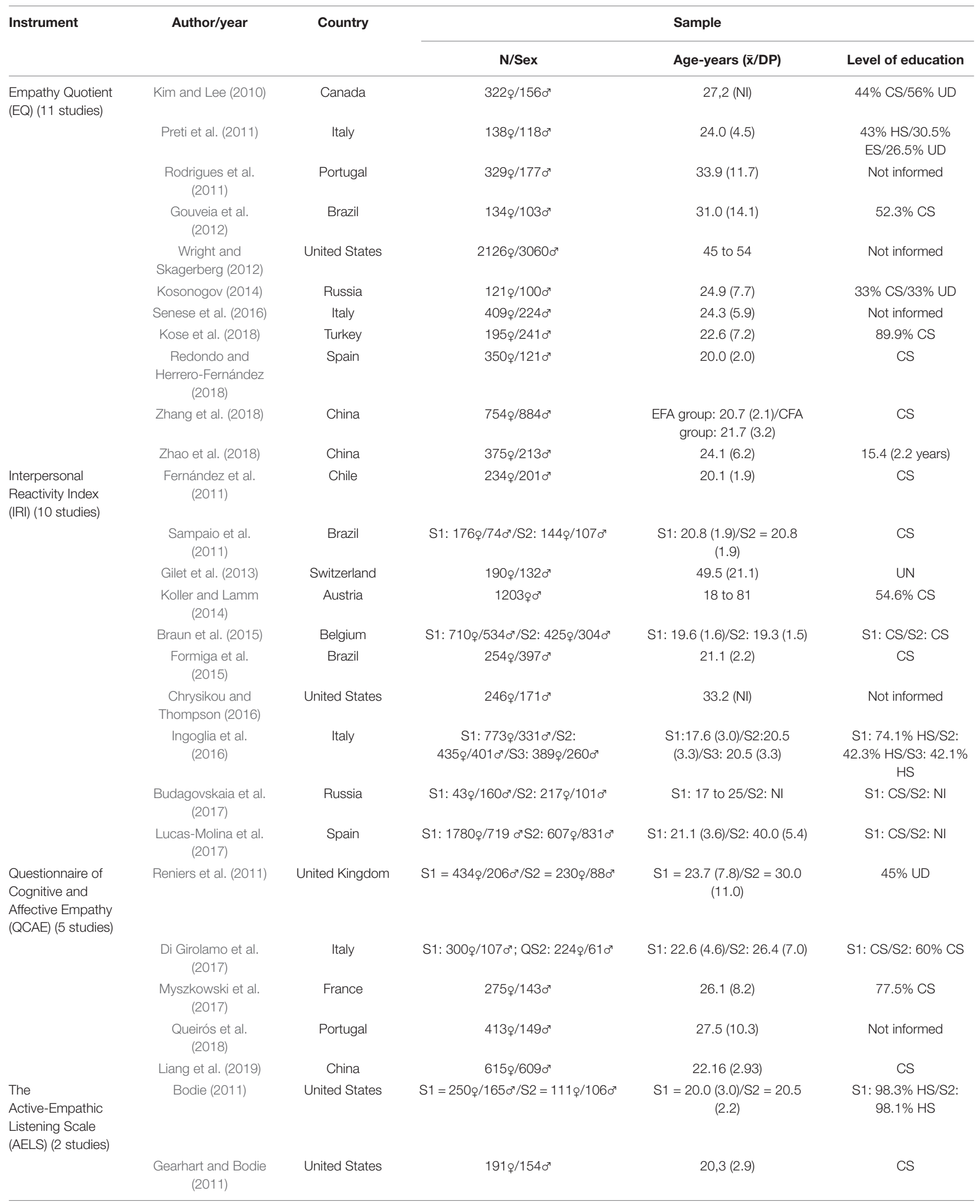


TABLE 2 | (Continued)

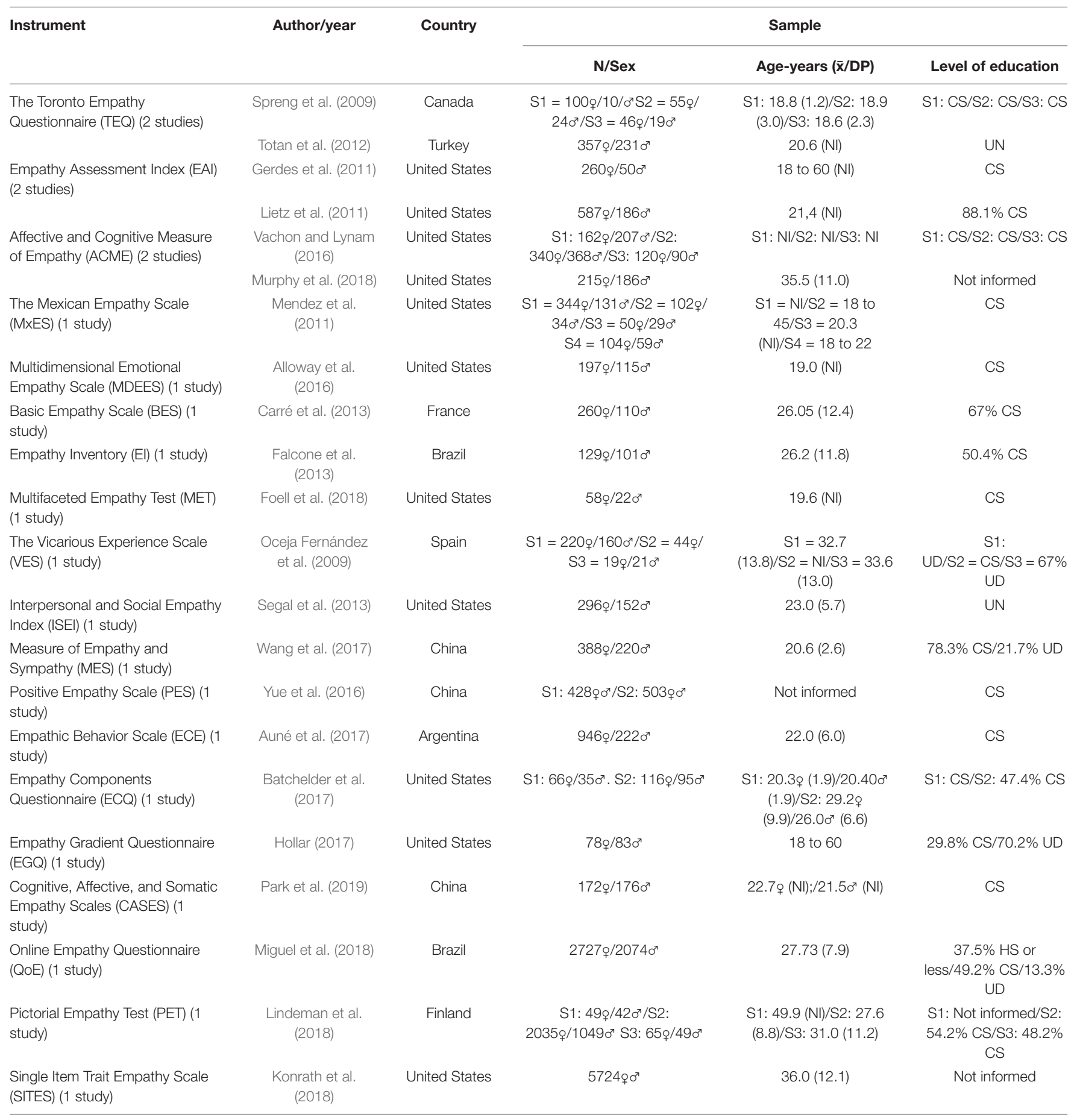

CS, college students; ES, elementary school; HS, high school; NS, non-student; UD, university degree o, women; ơ, men; S, sample.

addressed young/middle-aged adults (median $=24.0$ ); with varied educational levels (college students and individuals with a university degree $=67.3 \%$ ). As for the countries of origin, European countries predominated $(n=20)$, followed by North American countries $(n=17)$, Asian $(n=6)$, and South American countries $(n=7)$.
The instruments psychometric proprieties were assessed according to the parameters proposed by Andresen (2000). The results of which are presented in Tables 3, 4 and Supplementary Material 2 present raw data concerning these indicators based on reliability and validity criteria (construct and criterion). 
TABLE 3 | Analysis of psychometric qualities by the different instruments, according to the studies analyzed $(n=50)$ - Part A.

\begin{tabular}{|c|c|c|c|c|c|c|c|c|}
\hline Instrument & Study & $\begin{array}{l}\text { Norms/standard } \\
\text { values }\end{array}$ & $\begin{array}{l}\text { Measurement } \\
\text { model }\end{array}$ & $\begin{array}{l}\text { Item/Instrument } \\
\text { Bias }\end{array}$ & $\begin{array}{c}\text { Burden } \\
(1 / 2)\end{array}$ & Reliability & $\begin{array}{l}\text { Alternative/accessible } \\
\text { forms }\end{array}$ & $\begin{array}{l}\text { Culture/Language } \\
\text { adaptation }\end{array}$ \\
\hline \multirow[t]{11}{*}{ Empathy Quotient (EQ) } & Kim and Lee (2010) EQ-40 & B & A & Face validity: B & A/A & $\begin{array}{l}\text { Internal consistency: B } \\
\text { Test-retest: A }\end{array}$ & C & B (Korean) \\
\hline & Preti et al. (2011) EQ-60 & B & Unvalued & Not applicable & A/A & $\begin{array}{l}\text { Internal consistency: B } \\
\text { Test-retest:A }\end{array}$ & C & B (Italian) \\
\hline & Rodrigues et al. (2011) EQ-22 & Unvalued & Unvalued & Face validity: A & A/A & Internal consistency: A & C & B (Portuguese) \\
\hline & Gouveia et al. (2012) EQ-15 & Unvalued & Unvalued & Face validity: $\mathrm{A}$ & A/A & $\begin{array}{l}\text { Internal consistency: C } \\
\text { Composite reliability: B }\end{array}$ & c & B (Brazilian version) \\
\hline & $\begin{array}{l}\text { Wright and Skagerberg (2012) } \\
\text { EQ-40 }\end{array}$ & B & Unvalued & $\begin{array}{l}\text { Change version: } \mathrm{A} \\
\text { Unchanged version: } \mathrm{B}\end{array}$ & A/A & $\begin{array}{l}\text { Internal consistency changed } \\
\text { version: A } \\
\text { unchanged version: A }\end{array}$ & $\begin{array}{c}\text { B } \\
\text { computerized }\end{array}$ & Not applicable \\
\hline & Kosonogov (2014) EQ-60 & B & A & Face validity: C & A/A & $\begin{array}{c}\text { Internal consistency - EQ-60 - } 1 \\
\text { Factor: A } \\
\text { EQ-29 - } 3 \text { factors: A } \\
\text { EQ-28 - Lawrence et al. } \\
\text { model - } 3 \text { factors: B } \\
\text { EQ-21 - 3 factors: B } \\
\text { EQ-15 - } 3 \text { factors: B } \\
\text { EQ-15 - Muncer and Ling } \\
\text { model - 3 factors: C } \\
\text { Test-retest: EQ-60: A }\end{array}$ & c & B (Russian version) \\
\hline & Senese et al. (2016) EQ-40 & B & Unvalued & Not applicable & A/A & Internal consistency: B & B computerized & B (Italian version) \\
\hline & Kose et al. (2018) EQ-60 & B & Unvalued & Face validity: B & A/A & $\begin{array}{l}\text { Internal consistency: B } \\
\text { Test-retest: A }\end{array}$ & c & B (Turkish version) \\
\hline & $\begin{array}{l}\text { Redondo and } \\
\text { Herrero-Fernández (2018) } \\
\text { EQ-60 }\end{array}$ & Unvalued & Unvalued & Not applicable & $\mathrm{A} / \mathrm{A}$ & Unvalued & c & B (Spanish version) \\
\hline & Zhang et al. (2018) EQ-60 & B & Unvalued & Face validity: $\mathrm{A}$ & A/A & $\begin{array}{l}\text { Internal consistency: B } \\
\text { Test-retest: A }\end{array}$ & c & B (Chinese version) \\
\hline & Zhao et al. (2018) EQ-60 & B & Unvalued & Face validity: A & A/A & $\begin{array}{l}\text { Internal consistency: } \\
\text { EQ-40: A/EQ-15: A } \\
\text { Test-retest: } \\
\text { EQ-40: A/EQ-15:B }\end{array}$ & B computerized & B (Chinese version) \\
\hline \multirow[t]{10}{*}{ Interpersonal Reactivity Index (IRI) } & Fernández et al. (2011) IRI-28 & B & Unvalued & Face validity: B & A/A & $\begin{array}{l}\text { Internal consistency: B } \\
\text { Test-retest: A }\end{array}$ & c & B (Spanish version) \\
\hline & Sampaio et al. (2011) IRI-28 & B & Unvalued & Face validity: B & A/A & Internal consistency: B & C & B (Brazilian version) \\
\hline & Gilet et al. (2013) IRI-28 & B & Unvalued & Face validity: $\mathrm{A}$ & A/A & $\begin{array}{l}\text { Internal consistency: B } \\
\text { Test-retest: A }\end{array}$ & c & B (French version) \\
\hline & Koller and Lamm (2014) IRI-28 & Unvalued & A & B & A/A & Internal consistency: B & c & B (German) \\
\hline & Braun et al. (2015) IRI-28 & B & Unvalued & Face validity: B & A/A & Internal consistency: B & c & B (French version) \\
\hline & Formiga et al. (2015) IRI-26 & Unvalued & Unvalued & Not applicable & A/A & Internal consistency:B & C & B (Brazilian version) \\
\hline & $\begin{array}{l}\text { Chrysikou and Thompson } \\
\text { (2016) IRI-28 }\end{array}$ & B & Unvalued & Not applicable & A/A & Unvalued & B computerized & Not applicable \\
\hline & $\begin{array}{l}\text { Ingoglia et al. (2016) } \\
\text { IRI-28/RI-16 }\end{array}$ & B & A & Not applicable & A/A & $\begin{array}{l}\text { Internal consistency - } \\
\quad \mid \mathrm{RI}-28 / \mathrm{RI}-16: \mathrm{B}\end{array}$ & c & Not applicable \\
\hline & $\begin{array}{l}\text { Budagovskaia et al. (2017) } \\
\text { IRI-28 }\end{array}$ & B & Unvalued & Face validity: $\mathrm{A}$ & A/A & Internal consistency: B & c & B (Russian version) \\
\hline & Lucas-Molina et al. (2017) IRI-28 & Unvalued & Unvalued & Not applicable & A/A & Internal consistency: B & c & B (Spanish) \\
\hline \multirow{2}{*}{$\begin{array}{l}\text { Questionnaire of Cognitive and Affective } \\
\text { Empathy (QCAE) }\end{array}$} & Reniers et al. (2011) & Unvalued & Unvalued & Face validity: B & $\mathrm{A} / \mathrm{A}$ & Internal consistency: B & c & Not applicable \\
\hline & Di Girolamo et al. (2017) & B & Unvalued & Not applicable & A/A & $\begin{array}{l}\text { Internal consistency: Paper-pencil } \\
\text { and Online } \\
\text { total: A/subscales: B }\end{array}$ & A paper-pencil & Not applicable \\
\hline
\end{tabular}


TABLE 3 | (Continued)

\begin{tabular}{|c|c|c|c|c|c|c|c|c|}
\hline Instrument & Study & $\begin{array}{l}\text { Norms/standard } \\
\text { values }\end{array}$ & $\begin{array}{l}\text { Measurement } \\
\text { model }\end{array}$ & $\begin{array}{l}\text { Item/Instrument } \\
\text { Bias }\end{array}$ & $\begin{array}{l}\text { Burden } \\
(1 / 2)\end{array}$ & Reliability & $\begin{array}{l}\text { Alternative/accessible } \\
\text { forms }\end{array}$ & $\begin{array}{l}\text { Culture/Language } \\
\text { adaptation }\end{array}$ \\
\hline & Myszkowski et al. (2017) & Unvalued & Unvalued & Face validity: B & A/A & Internal consistency: B & $\mathrm{c}$ & B (French version) \\
\hline & Queirós et al. (2018) & B & Unvalued & Face validity: B & A/A & $\begin{array}{l}\text { Internal consistency } \\
\text { total: A/subscales: B }\end{array}$ & c & B (Portuguese version) \\
\hline & Liang et al. (2019) & B & Unvalued & Face validity: A & A/A & $\begin{array}{l}\text { Internal consistency } \\
\text { total: A/subscales: B } \\
\text { Test-retest } \\
\text { total: A/subscales: B }\end{array}$ & c & B (Chinese version) \\
\hline \multirow[t]{2}{*}{ The Active-Empathic Listening Scale (AELS) } & Bodie (2011) & Unvalued & Unvalued & Face validity: C & A/A & $\begin{array}{l}\text { Internal consistency } \\
\text { total: A/subscales: B }\end{array}$ & B other-report & Not applicable \\
\hline & Gearhart and Bodie (2011) & Unvalued & Unvalued & Not applicable & A/A & Not applicable & c & Not applicable \\
\hline \multirow[t]{2}{*}{ The Toronto Empathy Questionnaire (TEQ) } & Spreng et al. (2009) & B & Unvalued & Face validity: C & A/A & Internal consistency: A & c & Not applicable \\
\hline & Totan et al. (2012) & Unvalued & Unvalued & Face validity: B & A/A & $\begin{array}{l}\text { Internal consistency: B } \\
\text { Test-retest: B }\end{array}$ & c & B (Turkish version) \\
\hline \multirow{2}{*}{$\begin{array}{l}\text { Affective and Cognitive Measure of } \\
\text { Empathy (ACME) }\end{array}$} & Vachon and Lynam (2016) & B & Unvalued & Face validity: C & A/A & Internal consistency: A & c & Not applicable \\
\hline & Murphy et al. (2018) & Unvalued & Unvalued & Not applicable & A/A & Not applicable & c & Not applicable \\
\hline \multirow[t]{2}{*}{ Empathy Assessment Index (EAl) } & Gerdes et al. (2011) & Unvalued & Unvalued & Face validity:A & A/A & $\begin{array}{l}\text { Internal consistency: A } \\
\text { Test-retest: B }\end{array}$ & c & Not applicable \\
\hline & Lietz et al. (2011) & Unvalued & Unvalued & Not applicable & A/A & $\begin{array}{l}\text { Internal consistency: B } \\
\text { Test-retest: B }\end{array}$ & c & Not applicable \\
\hline Measure of Empathy and Sympathy (MES) & Wang et al. (2017) & Unvalued & Unvalued & Not applicable & A/A & $\begin{array}{l}\text { Internal consistency } \\
\text { total: B/subscales: B }\end{array}$ & c & B (Adult version) \\
\hline $\begin{array}{l}\text { Multidimensional } \\
\text { Emotional Empathy Scale (MDEES) }\end{array}$ & Alloway et al. (2016) & B & Unvalued & Not applicable & A/A & Internal consistency: A & c & Not applicable \\
\hline Basic Empathy Scale (BES) & Carré et al. (2013) & B & Unvalued & Not applicable & A/A & $\begin{array}{c}\text { Internal consistency: } \\
2 \text { Factors:B/3 Factors: B } \\
\text { Test-retest: } \\
2 \text { factors: B/3 factors: B }\end{array}$ & c & B (Adult version) \\
\hline Empathy Inventory (EI) & Falcone et al. (2013) & Unvalued & Unvalued & Not applicable & A/A & Not applicable & c & Not applicable \\
\hline Multifaceted Empathy Test (MET) & Foell et al. (2018) & Unvalued & Unvalued & Face validity: A & $\mathrm{B} / \mathrm{B}$ & $\begin{array}{l}\text { Internal consistency Cognitive } \\
\text { empathy (before/after reduction): C } \\
\text { Emotional empathy: A }\end{array}$ & c & B (English version) \\
\hline The Vicarious Experience Scale (VES) & Oceja Fernández et al. (2009) & Unvalued & Unvalued & Face validity: C & A/A & Internal consistency: B & c & Not applicable \\
\hline $\begin{array}{l}\text { Interpersonal and Social Empathy Index } \\
\text { (ISEI) }\end{array}$ & Segal et al. (2013) & Unvalued & Unvalued & Face validity: A & A/A & $\begin{array}{l}\text { Internal consistency } \\
\text { total:A/subscales: B }\end{array}$ & c & Not applicable \\
\hline The Mexican Empathy Scale (MxES) & Mendez et al. (2011) & $\mathrm{B}$ & Unvalued & Face validity: A & A/A & Internal consistency: B & c & B (English version) \\
\hline Positive Empathy Scale (PES) & Yue et al. (2016) & Unvalued & Unvalued & Face validity: A & A/A & $\begin{array}{l}\text { Internal consistency: A } \\
\text { Test-retest:A }\end{array}$ & c & B (Chinese version) \\
\hline Empathic Behavior scale (ECE) & Auné et al. (2017) & Unvalued & Unvalued & Face validity: C & A/A & Internal consistency: A & c & Not applicable \\
\hline Empathy Components Questionnaire (ECQ) & Batchelder et al. (2017) & B & Unvalued & Face validity: A & A/A & Internal consistency: B & c & Not applicable \\
\hline Empathy Gradient Questionnaire (EGQ) & Hollar (2017) & B & Unvalued & Face validity: C & A/A & Internal consistency: A & c & Not applicable \\
\hline $\begin{array}{l}\text { Cognitive, Affective, and Somatic Empathy } \\
\text { Scales (CASES) }\end{array}$ & Park et al. (2019) & Unvalued & Unvalued & Face validity: $B$ & $\mathrm{~A} / \mathrm{A}$ & $\begin{array}{l}\text { Internal consistency } \\
1 \text { and } 2 \text { Factors: } \mathrm{A} \\
3 \text { factors: } \mathrm{B}\end{array}$ & c & $\begin{array}{l}B \text { (Chinese version for } \\
\text { adults) }\end{array}$ \\
\hline Online Empathy Questionnaire (QoE) & Miguel et al. (2018) & Unvalued & Unvalued & Face validity: C & A/A & $\begin{array}{l}\text { Internal consistency } \\
\text { total: A/subscales: B } \\
\text { Test-retest: A }\end{array}$ & c & Not applicable \\
\hline Pictorial Empathy Test (PET) & Lindeman et al. (2018) & Unvalued & Unvalued & Face validity: C & A/A & Internal consistency: A & c & Not applicable \\
\hline Single Item Trait Empathy Scale (SITES) & Konrath et al. (2018) & B & Unvalued & Face validity: C & A/A & Test-retest: B & c & Not applicable \\
\hline
\end{tabular}


TABLE 4 | Analysis of psychometric qualities by the different instruments according to the studies analyzed $(N=50)$ - Part B.

\begin{tabular}{|c|c|c|c|c|c|}
\hline Instrument & Study & Factor Structure & Convergent/Discriminant & Known groups & $\begin{array}{c}\text { Predictive/ } \\
\text { Responsiveness }\end{array}$ \\
\hline \multirow[t]{11}{*}{$\begin{array}{l}\text { Empathy Quotient } \\
\text { (EQ) }\end{array}$} & Kim and Lee (2010) EQ-40 & $\begin{array}{l}\text { CFA - EQ-40: } 3 \text { Factors Model: C } \\
\text { CFA - EQ-28: } 1 \text { Factor Model: C } \\
\text { CFA - EQ-28: Lawrence et al. - } 3 \text { Factors Model: B } \\
\text { CFA - EQ-15: Muncer and Ling - } 3 \text { Factors Model: A }\end{array}$ & Convergent: B & Gender: B & Not evaluated \\
\hline & Preti et al. (2011) EQ-60 & CFA - EQ-60: 3 Factors Model: B & $\begin{array}{l}\text { Convergent (related } \\
\text { construct): B } \\
\text { Discriminant: A }\end{array}$ & Gender: A & Not evaluated \\
\hline & $\begin{array}{l}\text { Rodrigues et al. (2011) } \\
\text { EQ-22 }\end{array}$ & EFA - EQ-22: 4 Factors Model: B & Discriminant: A & Not evaluated & Not evaluated \\
\hline & Gouveia et al. (2012) EQ-15 & $\begin{array}{l}\text { CFA - EQ-15: } 1 \text { Factor Model: C } \\
\text { CFA - EQ-15 - Muncer and Ling - } 3 \text { Factors Model: C }\end{array}$ & Not evaluated & $\begin{array}{l}\text { Age: A } \\
\text { Gender: A } \\
\text { Education: C }\end{array}$ & Not evaluated \\
\hline & $\begin{array}{l}\text { Wright and Skagerberg } \\
\text { (2012) EQ-40 }\end{array}$ & Not evaluated & $\begin{array}{l}\text { Convergent: B } \\
\text { Convergent (related } \\
\text { construct): B } \\
\text { Discriminant: A }\end{array}$ & Gender: A & Not evaluated \\
\hline & Kosonogov (2014) EQ-60 & $\begin{array}{l}\text { EFA: B } \\
\text { CFA - EQ-60: } 1 \text { Factor Model: C } \\
\text { CFA - EQ-29 - } 3 \text { Factors Model: C } \\
\text { CFA - EQ-28 - Lawrence et al. - } 3 \text { Factors Model: C } \\
\text { CFA - EQ-21 - } 3 \text { Factors Model: C } \\
\text { CFA - EQ-15 - } 3 \text { Factors Model: B } \\
\text { CFA - EQ-15 - Muncer and Ling - } 3 \text { Factors Model: B } \\
\text { CFA - EQ-14 - } 1 \text { Factor Model: A }\end{array}$ & Convergent: B & Gender: A & Not evaluated \\
\hline & Senese et al. (2016) EQ-40 & $\begin{array}{l}\text { CFA - EQ-40 - } 1 \text { Factor Model: C } \\
\text { CFA - EQ-28 - } 1 \text { Factor Model: A } \\
\text { CFA - EQ-28 - Lawrence et al. - } 3 \text { Factors Model: A } \\
\text { CFA - EQ-15 - } 1 \text { Factor Model: C } \\
\text { CFA - EQ-15 - Muncer and Ling - } 3 \text { Factors Model: A } \\
\text { Measurement invariance: gender }\end{array}$ & $\begin{array}{l}\text { Convergent: B } \\
\text { Convergent (related } \\
\text { construct): B } \\
\text { Discriminant: A }\end{array}$ & Gender: B & Not evaluated \\
\hline & Kose et al. (2018) EQ-60 & EFA: B & Convergent: C & Gender: A & Not evaluated \\
\hline & $\begin{array}{l}\text { Redondo and } \\
\text { Herrero-Fernández (2018) } \\
\text { EQ-60 }\end{array}$ & $\begin{array}{l}\text { CFA - EQ-28 - Lawrence et al. - } 3 \text { Factors Model: A } \\
\text { CFA - EQ-23 - } 3 \text { Factors Model (factor load > 0.20): A } \\
\text { CFA - EQ-23 - } 1 \text { Factor Model (factor load > 0.20): A } \\
\text { CFA - EQ-15 - Kim and Lee - } 3 \text { Factors Model: C } \\
\text { CFA - EQ-15 - } 1 \text { Factor Model: A }\end{array}$ & $\begin{array}{l}\text { Convergent: B } \\
\text { Convergent (related } \\
\text { construct): B }\end{array}$ & Gender: B & Not evaluated \\
\hline & Zhang et al. (2018) EQ-60 & CFA - EQ-29 - 4 Factors Model: A & Not evaluated & Gender: A & Not evaluated \\
\hline & Zhao et al. (2018) EQ-60 & $\begin{array}{l}\text { CFA - EQ-40 - } 1 \text { Factor Model: C } \\
\text { CFA - EQ-40 - Lawrence et al. - } 3 \text { Factors Model: C } \\
\text { CFA - EQ-22 - Wakabayashi et al. - } 1 \text { Factor Model: C } \\
\text { CFA - EQ-15 - Muncer and Ling - } 3 \text { Factors Model: C } \\
\text { CFA - EQ-26 - Alisson et al. - } 2 \text { Factors Model: A } \\
\text { CFA - EQ-15 - Modified Guan et al. - } 1 \text { Factor Model: A } \\
\text { CFA - EQ-15 - Guan et al. - } 1 \text { Factor Model: A }\end{array}$ & $\begin{array}{l}\text { Convergent: B } \\
\text { Convergent (related } \\
\text { construct): B }\end{array}$ & $\begin{array}{l}\text { EQ-40: gender: A } \\
\text { EQ-15: gender: C }\end{array}$ & Not evaluated \\
\hline \multirow[t]{9}{*}{$\begin{array}{l}\text { Interpersonal } \\
\text { Reactivity Index (IRI) }\end{array}$} & $\begin{array}{l}\text { Fernández et al. (2011) } \\
\text { IRI-28 }\end{array}$ & $\begin{array}{l}\text { CFA - Davis Original } 4 \text { Factors Model: C } \\
\text { CFA - Cliffordson } 2^{\circ} \text { order } 4 \text { Factors Model: C }\end{array}$ & $\begin{array}{l}\text { Convergent (related } \\
\text { construct): B }\end{array}$ & Gender: A & Not evaluated \\
\hline & Sampaio et al. (2011) IRI-28 & $\begin{array}{l}\text { EFA - } 26 \text { items - } 4 \text { Factors Model: B } \\
\text { CFA - } 1 \text { Factor Model: A } \\
\text { CFA - } 2 \text { Factors Model: A } \\
\text { CFA - Davis Original } 4 \text { Factors Model: A }\end{array}$ & Not evaluated & Gender: A & Not evaluated \\
\hline & Gilet et al. (2013) IRI-28 & $\begin{array}{l}\text { CFA - } 1 \text { Factor Model: C } \\
\text { CFA - } 2 \text { Factors Model: C } \\
\text { CFA - Davis Original } 4 \text { Factors Model: C }\end{array}$ & Convergent: B & $\begin{array}{l}\text { Gender: } \mathrm{A} \\
\text { age: } \mathrm{B}\end{array}$ & Not evaluated \\
\hline & $\begin{array}{l}\text { Koller and Lamm (2014) } \\
\text { IRI-28 }\end{array}$ & $\begin{array}{l}\text { CFA - } 3 \text { Factors Model: A } \\
\text { CFA - Davis Original } 4 \text { Factors Model: A } \\
\text { CFA - } 5 \text { Factors Model: A } \\
\text { Measurement invariance: gender and age }\end{array}$ & Not evaluated & Not evaluated & Not evaluated \\
\hline & Braun et al. (2015) IRI-28 & $\begin{array}{l}\text { Sample 1: EFA - IRI-15 - } 4 \text { Factors Model: B } \\
\text { CFA - IRI-15 - } 4 \text { Factors Model: A } \\
\text { CFA - Davis Original Model - } 4 \text { Factors: B } \\
\text { Sample 2: CFA - IRI-15 - } 4 \text { Factors Model: A } \\
\text { Measurement invariance: gender }\end{array}$ & $\begin{array}{l}\text { Convergent (related } \\
\text { construct): B }\end{array}$ & Gender: A & Not evaluated \\
\hline & Formiga et al. (2015) IRI-26 & $\begin{array}{l}\text { EFA - Ribeiro et al. - } 3 \text { Factors Model: A } \\
\text { EFA - Sampaio et al. } 4 \text { Factors Model: A }\end{array}$ & Not evaluated & Not evaluated & Not evaluated \\
\hline & $\begin{array}{l}\text { Chrysikou and Thompson } \\
\text { (2016) IRI-28 }\end{array}$ & $\begin{array}{l}\text { CFA - } 2 \text { Factors Model: C } \\
\text { CFA - Pulos et al. - } 3 \text { Factors Model: B } \\
\text { CFA - Davis Original } 4 \text { | Factors Model: B }\end{array}$ & Not evaluated & Gender: A & Not evaluated \\
\hline & $\begin{array}{l}\text { Ingoglia et al. (2016) IRI-28 } \\
\text { IRI-16 }\end{array}$ & $\begin{array}{l}\text { Sample 1: EFA - } 16 \text { items - } 4 \text { Factors Model: B } \\
\text { Sample 2: CFA - } 16 \text { items - } 4 \text { Factors Model: A } \\
\text { Sample 3: CFA - } 16 \text { items - } 4 \text { factors: A } \\
\text { Measurement invariance: gender and age }\end{array}$ & $\begin{array}{l}\text { Convergent (related } \\
\text { construct): A }\end{array}$ & Gender: A & Not evaluated \\
\hline & $\begin{array}{l}\text { Budagovskaia et al. (2017) } \\
\text { IRI-28 }\end{array}$ & EFA - Davis Original 4| Factors Model: C & $\begin{array}{c}\text { Convergent: A } \\
\text { Convergent (related } \\
\text { construct): B }\end{array}$ & Gender: A & Not evaluated \\
\hline
\end{tabular}


TABLE 4 | (Continued)

\begin{tabular}{|c|c|c|c|c|c|}
\hline Instrument & Study & Factor Structure & Convergent/Discriminant & Known groups & $\begin{array}{c}\text { Predictive/ } \\
\text { Responsiveness }\end{array}$ \\
\hline & $\begin{array}{l}\text { Lucas-Molina et al. } \\
\text { (2017) IRI-28 }\end{array}$ & $\begin{array}{l}\text { CFA - Davis Original } 4 \text { Factors Model: C } \\
\text { CFA - Hawk et al: } 4 \text { Factors 2nd order Model: C } \\
\text { Measurement invariance: gender }\end{array}$ & Not evaluated & Gender: B & Not evaluated \\
\hline \multirow{5}{*}{$\begin{array}{l}\text { Questionnaire of } \\
\text { Cognitive and } \\
\text { Affective Empathy } \\
\text { (QCAE) }\end{array}$} & Reniers et al. (2011) & $\begin{array}{l}\text { EFA: B } \\
\text { CFA - } 5 \text { Factors Model }-1 \text { st order: A } \\
\text { CFA - } 5 \text { Factors Model }-2 \text { nd order: A }\end{array}$ & $\begin{array}{l}\text { Convergent: A } \\
\text { Convergent (related } \\
\text { construct): B }\end{array}$ & Gender: A & Not evaluated \\
\hline & $\begin{array}{l}\text { Di Girolamo et al. } \\
\text { (2017) }\end{array}$ & $\begin{array}{l}\text { CFA - Paper-pencil scales dataset: } \\
1 \text { Factor Model: C } \\
\text { Reniers et al. Original } 5 \text { Factors Model - 1st order: A } \\
\text { Reniers et al. Original } 5 \text { Factors Model - 2nd order: A } \\
\text { CFA - Online dataset } \\
1 \text { Factor Model: C } \\
\text { Reniers et al. Original } 5 \text { Factors Model - } 1 \text { st order: A } \\
\text { Reniers et al. Original } 5 \text { Factors Model - 2nd order: A } \\
\text { Measurement invariance: paper-pencil x online version }\end{array}$ & $\begin{array}{c}\text { Convergent (related } \\
\text { construct): } \\
\text { Paper-pencil scales dataset: } \\
\text { B } \\
\text { Online dataset: B }\end{array}$ & Not evaluated & Not evaluated \\
\hline & $\begin{array}{l}\text { Myszkowski et al. } \\
(2017)\end{array}$ & $\begin{array}{l}\text { CFA - Reniers et al. Original } 5 \text { Factors Model: A } \\
\text { CFA - } 5 \text { Factors Model }-2 \text { nd order: A } \\
\text { CFA - } 5 \text { Factors Model ( } 1 \text { inequality constraint): A } \\
\text { CFA - } 5 \text { Orthogonal Factors Model: C } \\
\text { CFA - } 5 \text { Factors Model- } 2 \text { nd order ( } 1 \text { inequality constraint): C } \\
\text { CFA - } 1 \text { Factor Model: C } \\
\text { CFA - } 2 \text { Orthogonal Factors Model: C } \\
\text { CFA - } 2 \text { Correlated Factors Model: C } \\
\text { Measurement invariance: gender }\end{array}$ & Not evaluated & Not evaluated & Not evaluated \\
\hline & Queirós et al. (2018) & $\begin{array}{l}\text { CFA - Reniers et al. Original } 5 \text { Factors Model: B } \\
\text { CFA - } 5 \text { Factors Model - with } 2 \text { correlated 2nd order: B } \\
\text { Measurement invariance: gender }\end{array}$ & Not evaluated & Gender: A & Not evaluated \\
\hline & Liang et al. (2019) & $\begin{array}{l}\text { EFA: B } \\
\text { CFA - Reniers et al. Original } 5 \text { Factors Model: A } \\
\text { CFA - } 5 \text { Factors Model - } 2 \text { nd order: A } \\
\text { CFA - } 4 \text { Factors Model - } 1 \text { st order: A } \\
\text { CFA - } 4 \text { Factors Model - } 2 \text { nd order: A } \\
\text { Measurement invariance: gender }\end{array}$ & $\begin{array}{l}\text { Convergent: A } \\
\text { Convergent (related } \\
\text { construct): C }\end{array}$ & Gender: A & Not evaluated \\
\hline \multirow[t]{2}{*}{$\begin{array}{l}\text { The } \\
\text { Active-Empathic } \\
\text { Listening Scale } \\
\text { (AELS) }\end{array}$} & Bodie (2011) & $\begin{array}{l}\text { Sample 1: CFA - self-report - } 3 \text { Factors Model: A } \\
\text { Sample 2: CFA - other-report - } 3 \text { Factors Model: B } \\
\text { FIA - Self-report x Other-report - } 3 \text { Factors Model: A } \\
\text { Measurement invariance: self and other-report version } \\
\text { (1st-order factor: measurement weights; 2nd-order factor: } \\
\text { structural weights) }\end{array}$ & $\begin{array}{l}\text { Self-report: } \\
\text { Convergent: B } \\
\text { Convergent (related } \\
\text { construct): A } \\
\text { Other-report: } \\
\text { Convergent (related } \\
\text { construct): A }\end{array}$ & $\begin{array}{l}\text { Good/bad } \\
\text { listener:A }\end{array}$ & Not evaluated \\
\hline & $\begin{array}{l}\text { Gearhart and Bodie } \\
\text { (2011) }\end{array}$ & CFA - 3 Factors Model: A & $\begin{array}{l}\text { Convergent (related } \\
\text { construct): B }\end{array}$ & Not evaluated & Not evaluated \\
\hline \multirow{2}{*}{$\begin{array}{l}\text { The Toronto } \\
\text { Empathy } \\
\text { Questionnaire } \\
\text { (TEQ) }\end{array}$} & Spreng et al. (2009) & Sample 1/2: EFA: B & $\begin{array}{l}\text { Convergent: A } \\
\text { Convergent (related } \\
\text { construct): B }\end{array}$ & Gender: A & Not evaluated \\
\hline & Totan et al. (2012) & $\begin{array}{l}\text { EFA - TEQ-16: } 1 \text { Factor Model: B } \\
\text { EFA - TEQ-13: } 1 \text { Factor Model: C } \\
\text { CFA - TEQ-13: } 1 \text { Factor Model: A } \\
\text { CFA - TEQ-13: } 1 \text { Factor Model (with error covariances):A } \\
\text { CFA - TEQ-16: } 1 \text { Factor Model: A }\end{array}$ & Convergent: A & Gender: A & Not evaluated \\
\hline \multirow{2}{*}{$\begin{array}{l}\text { Affective and } \\
\text { Cognitive Measure } \\
\text { of Empathy } \\
\text { (ACME) }\end{array}$} & $\begin{array}{l}\text { Vachon and Lynam } \\
\text { (2016) }\end{array}$ & $\begin{array}{l}\text { Sample1/2: CFA - } 3 \text { Factors Model: A } \\
\text { Measurement invariance: gender }\end{array}$ & $\begin{array}{l}\text { Convergent: A } \\
\text { Convergent (related } \\
\text { construct): B }\end{array}$ & Not evaluated & Not evaluated \\
\hline & Murphy et al. (2018) & $\begin{array}{l}\text { CFA - } 3 \text { Factors Model: C } \\
\text { CFA - } 5 \text { Factors Model: A } \\
\text { ESEM - } 3 \text { Factors Model: A } \\
\text { ESEM - } 4 \text { Factors Model: A }\end{array}$ & $\begin{array}{c}\text { Convergent: A } \\
\text { Convergent (related } \\
\text { construct): A }\end{array}$ & Not evaluated & Not evaluated \\
\hline \multirow{2}{*}{$\begin{array}{l}\text { Empathy } \\
\text { Assessment Index } \\
\text { (EAl) }\end{array}$} & Gerdes et al. (2011) & EFA: B & Convergent: A & Not evaluated & Not evaluated \\
\hline & Lietz et al. (2011) & $\begin{array}{l}\text { 1st half of the sample } \\
\text { CFA - EAl- } 40-5 \text { Factors Model: C } \\
\text { CFA - EAl- } 24-5 \text { Factors Model: B } \\
\text { CFA - EAl- } 24-5 \text { Factors Model (with eight error covariance): } \\
\text { A } \\
\text { CFA - EAl- } 17-5 \text { Factors Model: A } \\
\text { CFA - EAl- } 17-5 \text { Factors Model - with correlated error: A } \\
\text { 2nd half of the sample } \\
\text { CFA - EAl- } 24-5 \text { Factors Model: C } \\
\text { CFA - EAl- } 17-5 \text { Factors Model: B } \\
\text { CFA - EAl-17 - } 5 \text { Factors Model (with eight error covariance): } \\
\text { A }\end{array}$ & $\begin{array}{l}\text { Convergent (related } \\
\text { construct): B }\end{array}$ & $\begin{array}{l}\text { Gender: B } \\
\text { Race: B } \\
\text { College majors: } \\
\text { B } \\
\text { Socioeconomic: } \\
\text { B }\end{array}$ & Not evaluated \\
\hline
\end{tabular}


TABLE 4 | (Continued)

\begin{tabular}{|c|c|c|c|c|c|}
\hline Instrument & Study & Factor Structure & Convergent/Discriminant & Known groups & $\begin{array}{c}\text { Predictive/ } \\
\text { Responsiveness }\end{array}$ \\
\hline $\begin{array}{l}\text { Measure of } \\
\text { Empathy and } \\
\text { Sympathy (MES) }\end{array}$ & Wang et al. (2017) & $\begin{array}{l}\text { EFA: A } \\
\text { CFA - } 3 \text { Factors Model: A }\end{array}$ & $\begin{array}{l}\text { Convergent: B } \\
\text { Convergent (related } \\
\text { construct): B }\end{array}$ & Gender: A & $\mathrm{B}$ \\
\hline $\begin{array}{l}\text { Multidimensional } \\
\text { Emotional } \\
\text { Empathy Scale } \\
\text { (MDEES) }\end{array}$ & Alloway et al. (2016) & EFA: B & $\begin{array}{l}\text { Convergent (related } \\
\text { construct): } \mathrm{C}\end{array}$ & Not evaluated & Not evaluated \\
\hline $\begin{array}{l}\text { Basic Empathy } \\
\text { Scale (BES) }\end{array}$ & Carré et al. (2013) & $\begin{array}{l}\text { CFA - BES - } 1 \text { Factor Model - } 20 \text { items: B } \\
\text { CFA - BES - } 2 \text { Factors Model - } 20 \text { items: A } \\
\text { CFA - BES - } 3 \text { Factors Model - } 20 \text { items: A } \\
\text { CFA - BES - } 3 \text { Factors Model - } 19 \text { items: A }\end{array}$ & $\begin{array}{l}\text { Convergent: A } \\
\text { Convergent (related } \\
\text { construct):C }\end{array}$ & Gender: A & Not evaluated \\
\hline $\begin{array}{l}\text { Empathy } \\
\text { Inventory (EI) }\end{array}$ & Falcone et al. (2013) & Not evaluated & Convergent: A & Not evaluated & Not evaluated \\
\hline $\begin{array}{l}\text { Multifaceted } \\
\text { Empathy Test } \\
\text { (MET) }\end{array}$ & Foell et al. (2018) & Not evaluated & $\begin{array}{l}\text { Convergent: B } \\
\text { Convergent (related } \\
\text { construct): B }\end{array}$ & Not evaluated & Not evaluated \\
\hline $\begin{array}{l}\text { The Vicarious } \\
\text { Experience Scale } \\
\text { (VES) }\end{array}$ & $\begin{array}{l}\text { Oceja Fernández et al. } \\
\text { (2009) }\end{array}$ & EFA: C & $\begin{array}{l}\text { Convergent: B } \\
\text { Discriminant: A }\end{array}$ & Not evaluated & $\mathrm{C}$ \\
\hline $\begin{array}{l}\text { Interpersonal and } \\
\text { Social Empathy } \\
\text { Index (ISEI) }\end{array}$ & Segal et al. (2013) & $\begin{array}{l}\text { EFA - } 1 \text { Factor Model: C } \\
\text { EFA - Uncorrelated } 4 \text { Factors Model: C } \\
\text { EFA - Correlated } 4 \text { Factors Model: B }\end{array}$ & Not evaluated & Not evaluated & Not evaluated \\
\hline $\begin{array}{l}\text { The Mexican } \\
\text { Empathy Scale } \\
\text { (MxES) }\end{array}$ & Mendez et al. (2011) & EFA: B & Convergent: A & Not evaluated & $\mathrm{C}$ \\
\hline $\begin{array}{l}\text { Positive Empathy } \\
\text { Scale (PES) }\end{array}$ & Yue et al. (2016) & $\begin{array}{l}\text { EFA: } 1 \text { Factor Model: A } \\
\text { CFA: } 1 \text { Factor Model: B }\end{array}$ & $\begin{array}{l}\text { Convergent: B } \\
\text { Convergent (related } \\
\text { construct): B } \\
\text { discriminant: A }\end{array}$ & Not evaluated & Not evaluated \\
\hline $\begin{array}{l}\text { Empathic } \\
\text { Behavior Scale } \\
\text { (ECE) }\end{array}$ & Auné et al. (2017) & EFA: A & $\begin{array}{l}\text { Convergent (related } \\
\text { construct): B }\end{array}$ & Gender: A & Not evaluated \\
\hline $\begin{array}{l}\text { Empathy } \\
\text { Components } \\
\text { Questionnaire } \\
\text { (ECQ) }\end{array}$ & Batchelder et al. (2017) & $\begin{array}{l}\text { Sample 1: EFA: B } \\
\text { Sample 2: CFA - Model 1: C } \\
\text { CFA - Model 2: C } \\
\text { CFA - Model 3: C } \\
\text { CFA - Model 4: B }\end{array}$ & Not evaluated & Gender: A & B \\
\hline $\begin{array}{l}\text { Empathy Gradient } \\
\text { Questionnaire } \\
\text { (EGQ) }\end{array}$ & Hollar (2017) & $\begin{array}{l}\text { CFA - } 1 \text { Factor Model: B } \\
\text { CFA - } 5 \text { Factors Model: A }\end{array}$ & Not evaluated & $\begin{array}{l}\text { Age: C } \\
\text { Gender: C } \\
\text { Race: C }\end{array}$ & Not evaluated \\
\hline $\begin{array}{l}\text { Cognitive, } \\
\text { Affective, and } \\
\text { Somatic Empathy } \\
\text { Scales (CASES) }\end{array}$ & Park et al. (2019) & $\begin{array}{l}\text { CFA - } 1 \text { Factor Model: B } \\
\text { CFA - } 2 \text { Factors Model: B } \\
\text { CFA - } 3 \text { Factors Model: B }\end{array}$ & $\begin{array}{l}\text { Convergent: A } \\
\text { Convergent (related } \\
\text { construct): A }\end{array}$ & Not evaluated & Not evaluated \\
\hline $\begin{array}{l}\text { Online Empathy } \\
\text { Questionnaire } \\
\text { (QoE) }\end{array}$ & Miguel et al. (2018) & Not evaluated & $\begin{array}{l}\text { Convergent (related } \\
\text { construct): A }\end{array}$ & Gender: A & Not evaluated \\
\hline $\begin{array}{l}\text { Pictorial Empathy } \\
\text { Test (PET) }\end{array}$ & Lindeman et al. (2018) & $\begin{array}{l}\text { CFA - } 1 \text { Factor Model: B } \\
\text { CFA - } 1 \text { Factor Model (with correlated error): A }\end{array}$ & $\begin{array}{l}\text { Convergent: B } \\
\text { Convergent (related } \\
\text { construct): B } \\
\text { discriminant: A }\end{array}$ & Gender: A & Not evaluated \\
\hline $\begin{array}{l}\text { Single Item Trait } \\
\text { Empathy Scale } \\
\text { (SITES) }\end{array}$ & Konrath et al. (2018) & Not evaluated & $\begin{array}{l}\text { Convergent: B } \\
\text { Convergent (related } \\
\text { construct): B }\end{array}$ & Gender: A & Not evaluated \\
\hline
\end{tabular}

CFA, confirmatory factor analysis; EFA, exploratory factor analysis; ESEM, exploratory structural equation model.

\section{Empathy Quotient}

Eleven studies (22.0\%) assessed the EQ's psychometric properties, six of which applied the instrument's complete version (60 items); three applied the 40-item version (filler items are removed); one study applied the 22-item version, and one the 15 -item version.

The Respondent burden criterion was considered satisfactory in all the studies and received grade A; all the versions were brief and well accepted by the target population. Administrative burden also received grade A because the EQ is easy to apply, score, and interpret. None of the studies presented specific normative indicators such as the $\mathrm{T}$ score or percentile distribution, only data concerning the mean score $(n=8)$, which resulted in grade $B$.

Regarding the Measurement model criterion, only Kim and Lee (2010; EQ-40) and Kosonogov (2014; EQ-60) presented kurtosis and asymmetry indicators to show the normality of data distribution. The remaining studies $(81.8 \%)$ did not report analyses with this purpose, revealing a weakness regarding this psychometric indicator.

Seven studies conducted the EQ cross-cultural adaptation into Korean, Portuguese (Portugal and Brazil), Russian, Turkish, and Chinese. Rodrigues et al. (2011), Gouveia et al. (2012), 
Zhang et al. (2018), and Zhao et al. (2018) obtained grade A in the Item/instrument bias criterion as they adopted the recommended guidelines for face validity, namely: translation, back translation, peer review, and pretest applied in the target population (Beaton et al., 2000). The Korean and Turkish versions (Kim and Lee, 2010; Kose et al., 2018) received grade B because these did not report a pretest. The Russian version (Kosonogov, 2014) obtained grade $\mathrm{C}$ because it did not report its procedures. Two other studies (Preti et al., 2011 and Senese et al., 2016) assessed the psychometric quality of the Italian version, using the version previously adapted by Baron-Cohen (2004), while Redondo and Herrero-Fernández (2018) analyzed the properties of the version previously adapted by Allison et al. (2011) into Spanish.

The internal consistency of the 60 -item version presented alpha values that ranged from 0.76 to 0.85 ; most obtained grade $\mathrm{B}(N=3)$. Even with a smaller number of items, the short versions maintained alpha values within a similar pattern (0.78 to 0.87 ). The cumulative alpha for total scale was 0.85 (IC95\%: $0.81-0.85)$ with moderate heterogeneity (I2 $=47.96 \%$; $Q=23.866, p=0.02)$. The funnel plot showed asymmetry (Egger's: $p<0.001$ ) (see Supplementary Material 3.1). Subgroup analyzes considering the instrument's different version indicate cumulative alpha values of 0.76 (CI95\%: 0.74-0.79; I2 = 7.52\%; $Q=2.166, p=0.34)$ for the 60-item version, 0.84 (CI95\%: $0.78-$ 0.89 ; I2 $=97.3 \% ; Q=37.044, p<0.001)$ for the 40 -item version, and 0.81 (CI95\%: 0.74-0.87; I2 = 96.4\%; $Q=49.668, p<0.001$ ) for the 15-item version.

The studies concerning temporal stability used the test-retest methodology $(N=6)$, with intervals between 1 and 4 weeks, and indicated excellent indexes (estimated average correlation coefficient: 0.89 (CI95\%: 0.83-0.94); I2 = 90.76\%; $Q=22.330$, $p=0.001$; Egger's: $p<0.001$ ) (see Supplementary Material 3.2).

Regarding convergent validity, the studies used the IRI, Self-assessed Empathizing, Questionnaire Measure of Emotional Empathy, and Quotient of Empathic Abilities as a reference and found weak to moderate correlations (most obtained grade B, with correlations between 0.30 and 0.60$)$. The estimated average correlation coefficient was 0.44 (CI95\%: 0.36-0.52; I2 = 87.8\%; $Q=77.398, p<0.001$; Egger's: $p=0.59$ ). Other studies adopted instruments that assess correlated constructs such as alexithymia, social desirability, autism symptoms, and theory of mind (predominance of grade B). The pooled correlation estimate for was 0.38 (CI95\%: 0.30-0.46; I2 = 93.8\%; $Q=194.799$, $p<0.001$; Egger's: $p<0.001$ ) (see Supplementary Material 3.3).

Divergent validity was mainly verified through instruments assessing specific psychiatric symptoms such as hallucination, delirium, hypomania, and systematization (an individual's ability to develop a system and analyze its variables, considering underlining rules that guide the system's behavior) $(N=4)$. The values found in these studies ranged from -0.33 and 0.24 and obtained grade A.

Still, in search of evidence of validity with other variables, most studies $(N=9)$ assessed differences between genders; women tended to rate higher in empathy than men, especially in the emotional factor (grade A predominated). Only Gouveia et al. (2012) investigated the EQ's scores concerning age and education. The authors verified that older age was accompanied by a decline in the EQ's emotional and social subscales. Education was associated with more frequent expressions of empathy in the instrument's cognitive, emotional, and social subscales. Only one study tested and verified the instrument's invariance regarding gender (Senese et al., 2016). Predictive validity/responsiveness was not investigated, revealing a gap in the literature.

The exploratory factor analyses presented models with a varied number of factors, which, however, did not explain the significant percentage of data variance $(<47.4 \%)$ (Hair et al., 2009). The well-established models proposed by Lawrence et al. (2004; 3 factors: Cognitive Empathy, Emotional Reactivity and Social Skills -28 items), and Muncer and Ling (2006; 3 factors: Cognitive Empathy, Emotional Reactivity, and Social Skills -15 items) were the ones most frequently tested in confirmatory analyses. The results signaled goodness of fit problems for most of the studies; only one-third was rated A in this regard. These two models' unidimensionality was also tested, presenting contradictory results, while the one-factor model for the 40and 60 -item versions was considered inadequate by the three studies assessing it.

Alternative three-factor models were tested for the 40- and 60 -item versions and did not found satisfactory goodness of fit indexes. Other models with a varied number of items and factors were also analyzed $(n=8)$. Those that obtained grade A included: 29-item/4-factor model (Zhang et al., 2018), 23-item/with one or 3-factor model (Redondo and Herrero-Fernández, 2018), 25-item/2-factor model, 15-item/one-factor model (Zhao et al., 2018), and 14-item/one-factor model (Kosonogov, 2014).

Regarding the instrument's format, Wright and Skagerberg (2012), Senese et al. (2016), and Zhao et al. (2018) tested the online format, the psychometric indicators of which were similar to the original version (pencil-and-paper format). However, the invariance between the versions was not objectively tested.

Note that Wright and Skagerberg (2012) tested an alternative version of the EQ-40, rewriting negative statements into positive to test the hypothesis that the original format was syntactically more complex and challenging. They verified that response time was shorter in the alternative format; however, the remaining psychometric findings were not the same as in the original version, so that the authors did not recommend its use.

\section{Interpersonal Reactivity Index}

Ten studies assessed the psychometric properties of the IRI's original and alternative versions (with 26,16 , and 15 items). The instrument was considered adequate in terms of Respondent burden and Administrative burden, either due to its brevity or ease of application and interpretation; grade A was obtained.

In terms of normative aspects, as previously observed with the EQ, $70 \%$ of the studies only presented data concerning the samples' mean scores and their respective standard deviations (grade B).

As for the Measurement Model criterion, only the studies by Koller and Lamm (2014), and Ingoglia et al. (2016) investigated this criterion. The first study assessed floor and ceiling effects, while the latter reported kurtosis and asymmetry indicators to verify the normality of the data distribution. The remaining 
(80\%) did not perform analysis with this purpose, so that there is a lack of studies analyzing items.

Half of the studies addressing the IRI presented its crosscultural adaptation into different languages (Spanish, Portuguese from Brazil, French, and Russian), in general presenting adequate methodology to assess face validity.

Reliability was verified through internal consistency and temporal stability. Moderate meta-analytic measures of internal consistency were found for each subscale (Empathic Concern: 0.70 (CI95\%: 0.67-0.72), $\mathrm{I} 2=87.02 \%, Q=78.399, p<0.001$, Egger's: $p=0.14$; Fantasy: 0.78 (CI95\%: 0.77-0.80), I $=77.75 \%$, $Q=49.355, p<0.001$, Egger's: $p=0.84$; Personal Distress: 0.72 (CI95\%: 0.70-0.74), I2 = 81,01\%, $Q=56.145, p<0.001$, Egger's: $p=0.93$; Perspective Taking: 0.69 (CI95\%: 0.67-0.71), $\mathrm{I} 2=79.51 \%, Q=67.167, p<0.001$, Egger's: $p=0.05$ see Supplementary Material 4.1). Test-retest reliability (8 to 12 weeks), even though restricted to two studies, presented excellent indexes $(>0.76)$.

Regarding validity, most studies focused on analyzing the scale's factorial structure, in which various models were tested using exploratory $(N=5)$ and confirmatory factor analyses $(N=8)$. Davis's (1983) original model (1983; 4 factors - Empathic Concern, Fantasy, Perspective Taking, and Personal Distress) was the most frequently tested model, though controversial, and in general unsatisfactory results were found. Four-factor alternative models were also investigated, with slightly superior results [e.g., Braun et al. (2015) -15 items and Formiga et al. (2015) -26 items]. Unidimensional and bidimensional models $(N=3)$ were assessed and also presented controversial results.

Convergent validity was performed with other three instruments to assess general empathy and instruments measuring correlated constructs such as positive and negative affect, self-esteem, anxiety, aggression, social desirability, social avoidance, emotional fragility, emotional intelligence, gender roles, and sense of identity. The correlations with correlated constructs tended to be higher (estimated average correlation coefficient: 0.45 (CI95\%: 0.34-0.56), I2 $=96.17 \%, Q=482.604$, $p<0.001$, Egger's: $p=0.02$ ) than the correlations with the construct itself [estimated average correlation coefficient: 0.31 (CI95\%: 0.22-0.40), I2 $=75.38 \%, Q=43.065, p<0.001$, Egger's: $p=0.99]$. The analysis of subgroups, considering each of the subscales individually, presented the following estimated mean values of correlation with other empathy measures: Empathic Concern: 0.46 (CI95\%: 0.27-0.66), Fantasy: 0.26 (CI95\%: 0.040.49), Personal Distress: 0.25 (CI95\%: 0.17-0.34) and Perspective Taking: 0.28 (CI95\%: $0.16-0.41)$ and Personal Distress: 0.25 (CI95\%: 0.17-0.34) (see Supplementary Material 4.2).

In most cases, validity based on other variables was assessed in terms of gender. However, Gilet et al. (2013) also investigated age differences, reporting that younger individuals tended to be more empathic than older individuals, especially in the Fantasy and Personal Distress subscales. The studies addressing the IRI did not investigate predictive validity or responsiveness. The only alternative to the instrument's original format (pencil-andpaper) was a computer version addressed by Chrysikou and Thompson (2016), comparing the equivalence between both (not invariance).
Invariance of the IRI model was verified for sex (Koller and Lamm, 2014; Braun et al., 2015; Ingoglia et al., 2016; LucasMolina et al., 2017) and age (Koller and Lamm, 2014; Ingoglia et al., 2016).

\section{Questionnaire of Cognitive and Affective Empathy}

The studies addressing the QCAE involved its original proposition (Reniers et al., 2011) and well-conducted crosscultural adaptations into French, Portuguese (Portugal), and Chinese, except for the fact that they did not use a pilot study to check for face validity (predominance of $B$ grade).

The QCAE was considered a brief instrument; its application takes no more than $15 \mathrm{~min}$. Scoring is manual and easy to interpret, rating the highest in terms of Respondent and Administrative burden quality. In general, these studies involved basic constructs of the Classic Psychometric Theory, while no data concerning standardization and item analysis were reported.

The alpha cumulative value for the total scale was 0.86 (CI95\%: $0.86-0.87 ; \mathrm{I} 2=0 \% ; Q=1.585, p=0.66$; Egger's: $p=0.64)$ and for the subscales it ranged from 0.61 (Peripheral Responsivity: CI95\%: $0.56-0.66$; I2 $=79.79 \% ; Q=27.311, p<0.001)$ to 0.87 (Perspective Taking: CI95\%: 0.85-0.88; I2 $=76.19 \% ; Q=18.741$, $p=0.002)$. For more details see Supplementary Material 5.1. Test-retest reliability $(r=0.76)$ was satisfactory; the latter was only reported by Liang et al. (2019).

Regarding validity, there was a predominance of studies investigating the scale's factorial structure $(n=5)$. The original study reports a five-factor structure (Perspective Taking, Emotion Contagion, Online Simulation, Peripheral Responsivity, and Proximal Responsivity) and few goodness-of-fit problems (grade A). Later, most studies (75\%) confirmed this structure and report adequate indexes (grade A). Some alternative models were investigated, and findings indicate acceptable goodness-offit for the QCAE's first and second-order four-factor structure (Liang et al., 2019), though the instrument's unidimensionality was not confirmed.

Regarding convergent validity with other empathy measures ( $n=2$ studies: Basic Empathy Scale and IRI), correlation ranged from 0.27 to 0.76 and obtained grade $\mathrm{A}$ (according to the criterion established, only one correlation above 0.60 was necessary to obtain the highest grade). Instruments were also used to assess correlated constructs (e.g., aggressiveness, alexithymia, impulsivity, interpersonal competence, psychopathy, and social anhedonia, among others). The coefficients in these studies were moderate, and most were graded. The estimated average correlation coefficient was 0.27 (CI95\%: $0.20-0.35$ ), $\mathrm{I} 2=92.12 \%, Q=183.846, p<0.001$, Egger's: $p=0.04-$ see Supplementary Material 5.2.

Studies addressing known groups analyses (gender) predominated $(N=3)$, reinforcing previous studies indicating that women have greater empathic ability than men. None of the studies addressing the QCAE investigated predictive validity or responsiveness.

One of the studies (Di Girolamo et al., 2017) assessed the equivalence between the pencil-and-paper and online 
formats, and both presented similar psychometric indexes and measurement invariance. Invariance was also verified for sex (Myszkowski et al., 2017; Liang et al., 2019).

\section{Active-Empathic Listening Scale}

The AELS was proposed in 2006 (Drollinger et al., 2006) for the specific context of the relationship established between seller and customer, but Bodie (2011) proposed its expanded use for interpersonal relationships in general. Even though Bodie (2011) reported that the original items had to be changed and adapted, no information concerning the items analysis was provided, so that the item/instrument bias criterion obtained grade $\mathrm{C}$.

Later, Gearhart and Bodie (2011) expanded the adapted version's psychometric studies, presenting well-assessed Respondent burden and Administrative burden (grade A). Only Bodie (2011) assessed internal consistency, and the coefficients for the instrument as a whole $(>0.86)$ were considered excellent (grade A).

From the factorial structure perspective, the three-factor model (Sensing, Processing, and Responding) was considered appropriate, specifically for the self-report version (grade A) (Bodie, 2011), which was later confirmed by Gearhart and Bodie (2011) (grade A).

The convergent validity indexes concerning the self-report version indicated that correlations for the correlated constructs (conversational adequacy, interaction implications, social skills; -0.16 to 0.67 - grade A) were more robust than for the general empathy construct ( 0.15 to 0.44$)$, which were considered moderate (grade B). Only correlations with correlated constructs (conversational adequacy, conversational effectiveness, nonverbal immediacy) were investigated for the other-report version, ranging from 0.15 to 0.75 , and considered adequate (grade A). The self-report and other-report versions evidenced invariance of measure.

Studies addressing validity with other variables investigated the relationship between empathy scores and whether an individual is considered a good or poor listener (having an active and emotional interaction or not). Good listeners tended to score higher in empathy. There are no studies addressing the AELS normative data or predictive validity or studies conducting cross-cultural adaptations.

\section{Toronto Empathy Questionnaire}

The Toronto Empathy Questionnaire (TEQ) was addressed by two studies between 2009 and 2019: the study that originally proposed it (Spreng et al., 2009) and the study of its crosscultural adaptation into Turkish (Totan et al., 2012). The process of developing TEQ was adequately described, but no pilot test was reported. A pilot test was implemented during its crosscultural adaptation, impeding the Item/instrument bias criterion from achieving the maximum grade. On the other hand, due to the instrument's ease of use and application, the Respondent burden and Administrative burden criteria were assessed and obtained grade A.

The TEQ's reliability was assessed using internal consistency ( $\alpha=0.79$ to 0.87 ; predominance of grade $\mathrm{A}$ ) and temporal stability $(0,73$; grade $B)$, which were adequate. In terms of factorial structure, Spreng et al. (2009) conducted two exploratory analyses, and a unidimensional structure was found in both, with factor loadings above 0.37 (grade B). Totan et al. (2012) replicated the TEQ's unifactorial structure but found problems in three specific items, which led them to retest the model after excluding these items. Both the 16-item and 13-item versions appeared satisfactory in the confirmatory analysis, and the shortest version was recommended.

The TEQ's convergent validity was verified by comparing other instruments measuring empathy and instruments assessing correlated constructs such as autism, ability to understand the mental states of others, and interpersonal perception. As expected, most correlations between TEQ and other instruments assessing empathy were higher ( 0.29 to 0.80 ; grade $A)$ than correlations with correlated constructs $(-0.33$ to 0.35 ; grade $\mathrm{B})$.

Finally, other evidence of validity was analyzed, having the gender as a reference, and showed that women scored higher than men. The TEQ studies did not investigate predictive validity or responsiveness and did not report alternative formats or transcultural adaptations.

\section{Empathy Assessment Index}

Gerdes et al. (2011) originally proposed the EAI, and Lietz et al. (2011) later assessed its psychometric properties. The authors described the process of instrument development and the theoretical conceptualization of each of the five factors composing it (Affective Response, Perspective Taking, SelfAwareness, Emotion Regulation and Empathic Attitudes); Grade A was granted to the Item/instrument bias criterion.

Like the remaining instruments presented thus far, the EAI was also considered easy to apply, and therefore, the Respondent burden and Administrative burden were rated with the highest grade. Its precision coefficients ranged from 0.30 to 0.83 , and temporal stability ranged from 0.59 to 0.85 ; the retest was applied with a 1-week interval (grade B).

The original study reports that the exploratory factor analysis indicated a 34-item and 6-factor structure (Empathetic Attitudes, Affective Response -happy, Perspective Taking, Affective Response -sad, Perspective Taking-Affective Response and Emotion Regulation), which explained $43.19 \%$ of the variance of data (grade B). Later, based on literature reviews and feedback provided by specific community groups and experts in empathy, Lietz et al. (2011) performed factor analyses for a new 48-item version, concluding that the model presenting the best goodness of fit was composed of 17 items and five factors (Affective Response, Perspective Taking, Self-Awareness, Emotion Regulation and Empathic Attitudes) (grade A).

Gerdes et al. (2011) verified the convergent validity of the 34-item version, comparing it with the IRI and the coefficients ranged from 0.48 to 0.75 ; grade A was obtained. Lietz et al. (2011) investigated convergent validity by comparing the EAI with correlated constructs, such as attention and cognitive emotion regulation. As expected, moderate coefficients were found $(-0.40$ to 0.51 ; grade $\mathrm{B})$.

Lietz et al. (2011) verified the EAI's validity concerning the different sociodemographic variables. The results suggest differences concerning race (Afro- and Latin-Americans tended 
to present greater empathetic behavior than Caucasians); educational background (social workers presented greater empathy than individuals from the criminal justice, sociology, education, or nursing fields); and the family of origin's socioeconomic status (poor/working-class individuals presented greater empathetic behavior than middle/high-class individuals). The studies addressing the EAI did not address predictive validity or responsiveness nor reported alternative formats.

\section{Affective and Cognitive Measure of Empathy}

Affective and cognitive measure of empathy (ACME) was proposed by Vachon and Lynam (2016), and later, new validity evidence was presented by Murphy et al. (2018). Vachon and Lynam did not report the procedures concerning the instrument's development so that the Item/instrument bias criterion obtained grade $\mathrm{C}$. However, the instrument presented the characteristics necessary to receive grade $\mathrm{A}$ in the Respondent burden and Administrative burden criteria. Note that Vachon and Lynam (2016) study obtained grade B in the Norms and standard values criteria because only the means and standard deviations of each of the instrument's scales were reported according to sex and race for the entire sample.

Its reliability was only investigated through the internal consistency method ( $>0.85$; Vachon and Lynam, 2016), indicating a gap concerning temporal stability indicators.

Investigations related to the instrument's factorial structure and convergent validity were found. Vachon and Lynam (2016) suggested a three-factor structure (Cognitive Empathy, Affective Resonance and Affective Dissonance) with satisfactory goodness-of-fit indexes (grade A) and invariance between genders. However, Murphy et al. (2018) were unable to replicate this model and obtained unsatisfactory goodness-offit indexes. Hence, they proposed a five-factor model (two factors based on the items' polarity - positive and negative items, in addition to Cognitive Empathy, Affective Resonance and Affective Dissonance factors), with presented criteria that obtained grade $\mathrm{A}$.

Convergent validity was verified in relation to IRI $(-0.24$ to 0.80 ) and the Basic Empathy Scale (0.40 to 0.65); these criteria obtained grade A. The results indicated grade A for both studies regarding the indexes concerning correlated constructs, such as aggressive behavior, externalizing disorders, and personality pathologies $(-0.83$ to 0.77$)$.

\section{Remaining Instruments}

Other 16 instruments were analyzed by single studies. Seven of these intended to propose new instruments, five intended to obtain additional validity evidence, and four conducted a cross-cultural adaptation of existing instruments.

Except for the MET, all the instruments obtained grade A in the Respondent burden and Administrative burden criteria because they were brief, well-accepted, easy to apply, score and interpret. MET obtained grade B in both items because it is composed of 80 items and its application/scoring requires specific software.
Analysis of the new instruments showed no specific normative indicators were reported for any of them (Norms, standard values, grade B). Reliability verified through internal consistency was investigated in $85.7 \%$ of the studies and, in general, presented satisfactory results (grade B). Temporal stability was verified in only $28.6 \%$ of the studies and presented positive results (grades A and B).

As for existing instruments, there is a lack of normative data (data reported by $33.3 \%$ of the studies were restricted to mean and standard deviation of the total score). Nonetheless, as verified in the studies previously presented, no specific comparison indicators were reported between groups (e.g., T score or percentile).

Note that only two studies addressing these new instruments (Segal et al., 2013 - Interpersonal and Social Empathy Index and Batchelder et al., 2017 - Empathy Components Questionnaire) reported information concerning how the instruments were developed and obtained grade A. Convergent $(n=7)$, factor $(n=6)$, discriminant $(n=5)$, and predictive $(n=2)$ analyses were performed to investigate the instruments' validity. Regarding the factorial structure, both the instruments proposed before 2009 and those proposed after 2009 obtained grade B, showing that this group of instruments' factorial structures was confirmed with a few goodness-of-fit problems.

In general, the quality of the results concerning convergent validity was considered moderate (grade B). Correlations with other instruments measuring empathy were similar to the correlations found with instruments measuring correlated constructs. Validity studies with other variables were also restricted to the investigation of gender, corroborating the findings reported in the literature; that is, women tend to be more empathic than men. Hollar (2017) expanded the variables of interest (age and ethnicity) but obtained no satisfactory results.

Among this group of studies, Oceja Fernández et al. (2009) investigated predictive validity concerning the Vicarious Experience Scale; the Sympathy and Vicarious Distress subscales did not present satisfactory indexes for the prediction of elicited empathy and personal anguish. Batchelder et al. (2017), in turn, report the predictive ability of the Empathy Components Questionnaire concerning the scores obtained in the Social Interests Index (grade B).

Regarding this group of instruments, note that the Pictorial Empathy Test (Lindeman et al., 2018) differs from the remaining. It presents higher ecological validity because it is composed of images of people, while the Single Item Trait Empathy Scale stands out because it is composed of a single item. In general, both presented satisfactory psychometric properties.

As for cross-cultural studies, in general, face validity procedures were in line with the guidelines recommended by Beaton et al. (2000), and most (75\%) obtained grade A. These studies' psychometric properties were considered satisfactory, while the Culture/language adaptations item obtained grade $\mathrm{B}$.

The instruments' reliability (internal consistency and temporal stability) was considered acceptable in most studies (grades $A$ and B). However, the cross-cultural study addressing the MET obtained indexes below the expected for the instrument's cognitive factor, even after decreasing the scale's number of items. 
Six studies investigated the instruments' factorial structure. The results showed acceptable indexes for the Measure of Empathy and Sympathy, Multidimensional Emotional Empathy Scale, Basic Empathy Scale, The Mexican Empathy Scale, Positive Empathy Scale, and Cognitive, Affective, and Somatic Empathy Scales.

Among this set of studies, Park et al. (2019) conducted a cross-cultural adaptation of the Cognitive, Affective, and Somatic Empathy Scales. This instrument presents a specific scale to assess somatic empathy, which, according to the authors, can be defined as a tendency to imitate and automatically synchronize other peoples' facial expressions, vocalizations, behaviors, and movements. Only this instrument presented this measure. In general, its psychometric qualities were considered satisfactory.

\section{DISCUSSION}

This review compiled the psychometric findings of 23 instruments available in the literature to assess empathy in the last 10 years. In general, the findings concerning the existent instruments [reliability generalization meta-analyses (Cronbach's alpha) with values between 0.61 and 0.86 ] reinforced previous indicators of adequate reliability (e.g., EQ: Baron-Cohen and Wheelwright, 2004; Lawrence et al., 2004; IRI: Davis, 1980; Cliffordson, 2002; AELS: Drollinger et al., 2006; MxES: DíazLoving et al., 1986; MDEES: Caruso and Mayer, 1998; BES: Jolliffe and Farrington, 2006; EI: Falcone et al., 2008; MET: Dziobek et al., 2008).

On the other hand, the results indicated problems concerning convergent validity and factorial structure, making little progress in the solution and discussion of these impasses, e.g., a low to moderate correlation was found, especially between the EQ, IRI and QCAE and other instruments assessing the empathy construct (meta-analytic measures of correlation between 0.31 and 0.44), while similar or higher values were found when correlating these with different correlated constructs (metaanalytic measures of correlation between 0.27 and 0.45), indicating that the instruments' clinical validity was greater than theoretical validity. Studies published before the period addressed in this review also indicated these limits concerning convergent validity (e.g., EQ vs. IRI: Lawrence et al., 2004; De Corte et al., 2007; IRI vs. Hogan Empathy Scale: Davis, 1983; AELS vs. IRI: Drollinger et al., 2006; BES vs. IRI: Jolliffe and Farrington, 2006; MET vs. IRI: Dziobek et al., 2008) and factorial structure (e.g., EQ: Lawrence et al., 2004; Muncer and Ling, 2006; IRI: Siu and Shek, 2005; De Corte et al., 2007; BES: Jolliffe and Farrington, 2006; EI: Falcone et al., 2008).

It is important to note that most of the instruments analyzed here did not reach a consensus regarding the best factorial structure, considering that various models were tested. The results concerning the goodness of fit suggest problems related to both the base model (Comparative Fit Index and Tucker Lewis Index below the expected) and population covariance (Root Mean Square Error of Approximation above the expected) (Bentler, 1990; Hu and Bentler, 1999; Thompson, 2004). These divergences possibly reflect on the analyses of convergent validity with the different instruments measuring empathy, most of which obtained values within moderate limits.

We believe that controversies concerning the multidimensional nature of empathy (Murphy et al., 2018) reflect on the analyses, especially when the target instruments' subscales are more specifically analyzed. Murphy et al. (2018) widely discuss these aspects and note a lack of consensus regarding the empathy construct and how different authors adopt such a concept when developing instruments. These authors note there is greater consensus regarding the presence of affective and cognitive components; however, the analyses of the bifactor models assessed here (e.g., concerning the IRI) also failed to present satisfactory factor indexes. Given this lack of consensus, Surguladze and Bergen-Cico (2020) stress the need to reconsider and discuss this construct, considering its different dimensions and directly and indirectly related mechanisms.

In addition to what Murphy et al. (2018) state regarding lack of consensus, this review's findings indicate that some studies do not specify the conceptual model of empathy that grounded the development of the instruments and which would theoretically ground the empirical analysis of the instruments' internal structure, especially in second-order more complex models and with a varied number of factors. It was the case of both new instruments, proposed during the period covered by this review (for example: QCAE: Reniers et al., 2011; QoE: Miguel et al., 2018; TEQ: Spreng et al., 2009), and older instruments (EQ: Baron-Cohen and Wheelwright, 2004; IRI: Davis, 1983; MxES: Díaz-Loving et al., 1986; EI: Falcone et al., 2008). A lack of theoretical models to properly ground the empathy construct and its dimensions possibly explains the restrictions concerning structural validity and lack of convergence between the different instruments.

Regarding the statistical techniques used to investigate the instruments' structures, Marsh (2018) highlight that newer models, such as the Structural Equations Models (Gefen et al., 2000), can more deeply capture the complexity of the empathy construct and also resolve a series of problems encountered based on the CFA approach (e.g., restricted factor loadings) (Marsh, 2018). Nonetheless, most of the studies opted for adopting confirmatory and exploratory factor analyses so that future studies are needed to invest in these technologies and techniques of analysis. Note that studies based on the Item Response Theory (Pasquali, 2020) can contribute to this impasse, considering that the studies addressed here attempted to improve the factor model by removing specific items.

On the other hand, the construct's clinical/empirical validity seems to be unanimous. Even though the studies were conducted with non-clinical samples, associations with different correlated constructs are adequate and reinforce the relationship of empathy with different psychopathological and behavioral indicators (e.g., autism: Komeda et al., 2019, Post-traumatic stress disorder: Feldman et al., 2019, and Borderline personality disorder: Flasbeck et al., 2019). However, for these instruments to be used in a clinical setting, aspects related to predictive evidence, which remain scarce, need to be explored. In this context, normative studies, which were not the target of the psychometric studies addressed here, are also needed. 
Cross-cultural studies were an important focus of interest among researchers within this topic. These are relevant studies because they enable generating and/or reinforcing psychological theories that take the cultural context into account (Gomes et al., 2018). Additionally, these studies enable applying the same instrument among different individuals belonging to different contexts and facilitate understanding the similarities and characteristics these groups share (Borsa et al., 2012), which is essential, especially in clinical research.

In general, the results of cross-sectional studies addressing instruments reported psychometric qualities comparable to the original versions, though cultural invariance was not assessed for any of the target instruments. Investigating invariance between different cultural groups answering an instrument is essential to identify whether there are significant differences between scores, and if that is the case, verify whether differences are related to actual differences at a latent trait level or the instruments' parameters are not equivalent (Damásio et al., 2016).

Regarding new instruments, note that the same few added in terms of Respondent burden and Administrative burden, considering that these aspects, except for the MET, obtained grade A, though were little discriminant. These instruments also innovated little in terms of format and structure. Most were based on self-reported items rated on a Likert scale. The studies also do not seem to overcome the critical points mentioned earlier in psychometric terms.

Interpersonal reactivity index, and more recently, $\mathrm{EQ}$, have been widely used in different clinical studies and applied in different target populations (e.g., Feeser et al., 2015; Fitriyah et al., 2020). However, despite their popularity, they present weaknesses concerning structural validity and limitations regarding responsiveness, standardization, and bias.

The conclusion is that despite the diversity of the instruments available to assess empathy and many associated psychometric studies, limitations stand out, especially in terms of validity. Hence, as noted by previous reviews that evaluated specific instruments of empathy and/or their performance in specific populations (Hemmerdinger et al., 2007; Yu and Kirk, 2009; Hong and Han, 2020), no instrument can be currently appointed as the gold standard.

\section{REFERENCES}

Alcorta-Garza, A., San-Martín, M., Delgado-Bolton, R., Soler-González, J., Roig, H., and Vivanco, L. (2016). Cross-validation of the Spanish HP-version of the jefferson scale of empathy confirmed with some cross-cultural differences. Front. Psychol. 7:1002. doi: 10.3389/fpsyg.2016.01002

Allison, C., Baron-Cohen, S., Wheelwright, S. J., Stone, M. H., and Muncer, S. J. (2011). Psychometric analysis of the Empathy Quotient (EQ). Pers. Individ. Diff. 51, 829-835. doi: 10.1016/j.paid.2011.07.005

${ }^{*}$ Alloway, T. P., Copello, E., Loesch, M., Soares, C., Watkins, J., Miller, D., et al. (2016). Investigating the reliability and validity of the Multidimensional Emotional Empathy Scale. Measurement 90, 438-442. doi: 10.1016/j.measurement.2016.05.014

${ }^{*}$ Articles included in the review.
Therefore, this field of study needs to advance in conceptual and theoretical terms. Such an advance will enable the establishment of more robust models to be empirically reproduced by the instruments. Additionally, problems with the internal structure of various instruments can be minimized or resolved using more sophisticated techniques based on the analysis and refinement of items. Normative and predictive studies can improve the validity of evidence of existing studies, favoring greater clinical applicability. Complementary studies of invariance, testing the effect of cultures, and alternative forms of application (especially those using technological resources, such as online and computer applications) are desirable and can expand the reach of instruments. Regarding the proposition of new instruments, there seems to remain a need for instruments with alternative formats to minimize response bias, especially social desirability, a recurrent problem in self-report instruments.

\section{DATA AVAILABILITY STATEMENT}

The raw data supporting the conclusions of this article will be made available by the authors, without undue reservation.

\section{AUTHOR CONTRIBUTIONS}

Both authors listed have made a substantial, direct, and intellectual contribution to the work, and approved it for publication.

\section{FUNDING}

Funding for this study was provided by Coordination for the Improvement of Higher Education Personnel (Capes); National Council for Scientific and Technological Development (CNPq Productivity Research Fellows - Process No. 302601/2019-8).

\section{SUPPLEMENTARY MATERIAL}

The Supplementary Material for this article can be found online at: https://www.frontiersin.org/articles/10.3389/fpsyg. 2021.781346/full\#supplementary-material

Andresen, E. M. (2000). Criteria for assessing the tools of disability outcomes research. Arch. Phys. Med. Rehabil. 81, S15-S20.

*Auné, S., Facundo, A., and Attorresi, H. (2017). Psychometric properties of a test for assessing empathic behavior. Rev. Iberoamericana Diagn. Eval. Avaliação Psicol. 3, 47-56.

Baron-Cohen, S. (2003). The Essential Difference: Men, Women and the Extreme Male Brain. London: Penguin.

Baron-Cohen, S. (2004). Questione Di Cervello: La Differenza Essenziale Tra Donne E Uomini. Milano: Mondadori.

Baron-Cohen, S., and Wheelwright, S. (2004). The empathy quotient: an investigation of adults with Asperger syndrome or high functioning autism, and normal sex differences. J. Autism. Dev. Disord. 34, 163-175. doi: 10.1023/b: jadd.0000022607.19833.00 
*Batchelder, L., Brosnan, M., and Ashwin, C. (2017). The development and validation of the empathy components questionnaire (ECQ). PLoS One 12:e0169185. doi: 10.1371/journal.pone.0169185

Beaton, D. E., Bombardier, C., Guillemin, F., and Ferraz, M. B. (2000). Guidelines for the process of cross-cultural adaptation of self-report measures. Spine 25, 3186-3191. doi: 10.1097/00007632-200012150-0 0014

Bentler, P. M. (1990). Comparative fit indexes in structural models. Psychol. Bull. 107, 238-246. doi: 10.1037/0033-2909.107.2.238

${ }^{*}$ Bodie, G. D. (2011). The active-empathic listening scale (AELS): conceptualization and evidence of validity within the interpersonal domain. Commun. Q. 59, 277-295. doi: 10.1080/01463373.2011.583495

Bora, E., and Baysan, L. (2009). Psychometric features of Turkish version of empathy quotient in university students. Bull. Clin. Psychopharmacol. 19, $39-43$.

Borsa, J. C., Damásio, B. F., and Bandeira, D. R. (2012). Cross-cultural adaptation and validation of psychological instruments: some considerations. Paidéia 22, $423-432$.

BRASIL. Ministério da Saúde (2014). Diretrizes Metodológicas: elaboração de revisão sistemática e metanálise de ensaios clínicos randomizados. $1^{\text {a }}$ edição. Brasília: Editora do Ministério da Saúde.

*Braun, S., Rosseel, Y., Kempenaers, C., Loas, G., and Linkowski, P. (2015). Selfreport of empathy: a shortened french adaptation of the interpersonal reactivity index (IRI) using two large Belgian samples. Psychol. Rep. 117, 735-753. doi: 10.2466/08.02.PR0.117c23z6

${ }^{*}$ Budagovskaia, N. A., Dobrovskaia, S. V., and Kariagina, T. D. (2017). Adapting M. Davis's multifactor empathy questionnaire. J. Russian East Eur. Psychol. 54, 441-469. doi: 10.1080/10610405.2017.1448179

${ }^{*}$ Carré, A., Stefaniak, N., D’ambrosio, F., Bensalah, L., and Besche-Richard, C. (2013). The basic empathy scale in adults (BES-A): factor structure of a revised form. Psychol. Assess. 25:679. doi: 10.1037/a0032297

Caruso, D. R., and Mayer, J. D. (1998). A Measure of Emotional Empathy for Adolescents and Adults. Durham, NH: University of New Hampshire.

${ }^{*}$ Chrysikou, E. G., and Thompson, W. J. (2016). Assessing cognitive and affective empathy through the interpersonal reactivity index: an argument against a two-factor model. Assessment 23, 769-777. doi: 10.1177/107319111559 9055

${ }^{*}$ Cliffordson, C. (2002). The hierarchical structure of empathy: dimensional organization and relations to social functioning. Scand. J. Psychol. 43, 49-59. doi: $10.1111 / 1467-9450.00268$

Damásio, B. F., Valentini, F., Núñes-Rodriguez, S. I., Kliem, S., Koller, S. H., Hinz, A., et al. (2016). Is the general self-efficacy scale a reliable measure to be used in cross-cultural studies? Results from Brazil, Germany and Colombia. Span. J. Psychol. 19:E29. doi: 10.1017/sjp.2016.30

Davis, M. H. (1980). A multidimensional approach to individual differences in empathy. J. Pers. Soc. Psychol. 10:85. doi: 10.3389/fpsyg.2021.588934

Davis, M. H. (1983). Measuring individual differences in empathy: evidence for a multidimensional approach. J. Pers. Soc. Psychol. 44:113.

Davis, M. H. (2018). Empathy: A Social Psychological Approach. Abingdon: Routledge.

De Corte, K., Buysse, A., Verhofstadt, L. L., Roeyers, H., Ponnet, K., and Davis, M. H. (2007). Measuring empathic tendencies: reliability and validity of the dutch version of the interpersonal reactivity index. Psychol. Belgica 47, 235-260. doi: $10.5334 / \mathrm{pb}-47-4-235$

${ }^{*}$ Di Girolamo, M., Giromini, L., Winters, C. L., Serie, C. M., and de Ruiter, C. (2017). The questionnaire of cognitive and affective empathy: a comparison between paper-and-pencil versus online formats in italian samples. J. Pers. Assess. 101, 159-170. doi: 10.1080/00223891.2017.1389745

Díaz-Loving, R., Andrade Palos, P., and Nadelsticher, M. A. (1986). Desarrollo de la Escala Multidimensional de Empatía. Rev. Psicol. Soc. Pers. 1, 1-12. doi: 10.17081/psico.23.44.3581

Drollinger, T., Comer, L. B., and Warrington, P. T. (2006). Development and validation of the active empathetic listening scale. Psychol. Market. 23, 161-180. doi: 10.1002/mar.20105

Duckworth, A. L., and Kern, M. L. (2011). A meta-analysis of the convergent validity of self-control measures. J. Res. Pers. 45, 259-268.
Dziobek, I., Rogers, K., Fleck, S., Bahnemann, M., Heekeren, H. R., Wolf, O. T., et al. (2008). Dissociation of cognitive and emotional empathy in adults with Asperger syndrome using the Multifaceted Empathy Test (MET). J. Autism. Dev. Disord. 38, 464-473. doi: 10.1007/s10803-007-0486-x

Egger, M., Smith, G. D., Schneider, M., and Minder, C. (1997). Bias in meta-analysis detected by a simple, graphical test. BMJ 315, 629-634. doi: 10.1136/bmj.315. 7109.629

${ }^{*}$ Falcone, E. M. D. O., Ferreira, M. C., Luz, R. C. M. D., Fernandes, C. S., Faria, C. D. A., D’Augustin, J. F., et al. (2008). Construction of a Brazilian measure to evaluate empathy: the Empathy Inventory (EI). Avaliação Psicol. 7, 321-334.

Falcone, E. M. D. O., Pinho, V. D. D., Ferreira, M. C., Fernandes, C. D. S., D’Augustin, J. F., Krieger, S., et al. (2013). Validade convergente do Inventário de Empatia (IE). Psico-USF 18, 203-209.

Feeser, M., Fan, Y., Weigand, A., Hahn, A., Gärtner, M., Böker, H., et al. (2015). Oxytocin improves mentalizing-pronounced effects for individuals with attenuated ability to empathize. Psychoneuroendocrinology 53, 223-232. doi: 10.1016/j.psyneuen.2014.12.015

Feldman, R., Levy, Y., and Yirmiya, K. (2019). The neural basis of empathy and empathic behavior in the context of chronic trauma. Front. Psychiatry 10:562. doi: $10.3389 /$ fpsyt. 2019.00562

*Fernández, A. M., Dufey, M., and Kramp, U. (2011). Testing the psychometric properties of the Interpersonal Reactivity Index (IRI) in Chile. Eur. J. Psychol. Assess. 27, 179-185. doi: 10.1027/1015-5759/a00 0065

Fitriyah, F. K., Saputra, N., Dellarosa, M., and Afridah, W. (2020). Does spirituality correlate with students' empathy during covid-19 pandemic? the case study of Indonesian students. Counsel. Educ. Int. J. Counsel. Educ. 5:820.

Flasbeck, V., Enzi, B., and Brüne, M. (2019). Enhanced processing of painful emotions in patients with borderline personality disorder: a functional magnetic resonance imaging Study. Front. Psychiatry 10:357. doi: 10.3389/fpsyt. 2019.00357

${ }^{*}$ Foell, J., Brislin, S. J., Drislane, L. E., Dziobek, I., and Patrick, C. J. (2018). Creation and validation of an english-language version of the multifaceted empathy test (MET). J. Psychopathol. Behav. Assess. 40, 431-439. doi: 10.1007/s10862-0189664-8

${ }^{*}$ Formiga, N. S., Sampaio, L. R., and Guimarães, P. R. B. (2015). How many dimensions measure empathy? Empirical evidence multidimensional scale of interpresonal reactivity in brazilian. Eureka 12, 80.

${ }^{*}$ Gearhart, C. C., and Bodie, G. D. (2011). Active-empathic listening as a general social skill: evidence from bivariate and canonical correlations. Commun. Rep. 24, 86-98. doi: 10.1080/08934215.2011.610731

Gefen, D., Straub, D. W., and Boudreau, M. C. (2000). Structural equation modeling and regression: guidelines for research practice. Commun. AIS 4, 1-77. doi: 10.1080/10705511.2021.1963255

${ }^{*}$ Gerdes, K. E., Lietz, C. A., and Segal, E. A. (2011). Measuring empathy in the 21st century: development of an empathy index rooted in social cognitive neuroscience and social justice. Soc. Work Res. 35, 83-93. doi: 10.1093/swr/ 35.2 .83

${ }^{*}$ Gilet, A. L., Mella, N., Studer, J., Grühn, D., and Labouvie-Vief, G. (2013). Assessing dispositional empathy in adults: a french validation of the interpersonal reactivity index (IRI). Can. J. Behav. Sci. 45:42. doi: 10.1037/a003 0425

Giummarra, M. J., Fitzgibbon, B. M., Georgiou-Karistianis, N., Beukelman, M., Verdejo-Garcia, A., Blumberg, Z., et al. (2015). Affective, sensory and empathic sharing of another's pain: the empathy for pain scale. Eur. J. Pain 19, 807-816. doi: 10.1002/ejp.607

Gomes, L. B., Bossardi, C. N., Bolze, S. D. A., Bigras, M., Paquette, D., Crepaldi, M. A., et al. (2018). Cross-cultural research in developmental psychology: theoretical and methodological considerations. Arquivos Brasil. Psicol. 70, 260275. doi: $10.1002 /$ wcs. 1515

${ }^{*}$ Gouveia, V. V., Milfont, T. L., Gouveia, R. S., Neto, J. R., and Galvão, L. (2012). Brazilian-portuguese empathy quotient: evidences of its construct validity and reliability. Span. J. Psychol. 15, 777-782. doi: 10.5209/rev_sjop.2012.v15.n2. 38889 
Hair, J. F., Black, W. C., Babin, B. J., Anderson, R. E., and Tatham, R. L. (2009). Multivariate Data Analysis. Tucson, AZ: Bookman Editora.

Hedges, L., and Olkin, I. (1985). Statistical Methods for Meta-Analysis. London: Academic Press.

Hemmerdinger, J. M., Stoddart, S. D., and Lilford, R. J. (2007). A systematic review of tests of empathy in medicine. BMC Med. Educ. 7:24. doi: 10.1186/1472-69207-24

${ }^{*}$ Hollar, D. W. (2017). Psychometrics and assessment of an empathy distance gradient. J. Psychoeduc. Assess. 35, 377-390. doi: 10.1177/0734282915623882

Hong, H., and Han, A. (2020). A systematic review on empathy measurement tools for care professionals. Educ. Gerontol. 46, 72-83.

Hu, L. T., and Bentler, P. M. (1999). Cutoff criteria for fit indexes in covariance structure analysis: conventional criteria versus new alternatives. Struct. Equ. Model. 6, 1-55. doi: 10.1080/10705519909540118

${ }^{*}$ Ingoglia, S., Lo Coco, A., and Albiero, P. (2016). Development of a brief form of the interpersonal reactivity index (B-IRI). J. Pers. Assess. 98, 461-471. doi: 10.1080/00223891.2016.1149858

Innamorati, M., Ebisch, S. J., Saggino, A., and Gallese, V. (2015). The "Feeling with others" project: development of a new measure of trait empathy. Psicoterapia Cogn. Comportamentale 21, 261-263.

Jolliffe, D., and Farrington, D. P. (2006). Development and validation of the basic empathy scale. J. Adolesc. 29, 589-611. doi: 10.1016/j.adolescence.2005.08.010

${ }^{*}$ Kim, J., and Lee, S. J. (2010). Reliability and validity of the Korean version of the empathy quotient scale. Psychiatry Investig. 7:24.

${ }^{*}$ Koller, I., and Lamm, C. (2014). Item response model investigation of the (German) interpersonal reactivity index empathy questionnaire. Eur. J. Psychol. Assess. 31, 211-221.

Komeda, H., Kosaka, H., Fujioka, T., Jung, M., and Okazawa, H. (2019). Do individuals with autism spectrum disorders help other people with autism spectrum disorders? An investigation of empathy and helping motivation in adults with autism spectrum disorder. Front. Psychiatry 10:376. doi: 10.3389/ fpsyt.2019.00376

${ }^{*}$ Konrath, S., Meier, B. P., and Bushman, B. J. (2018). Development and validation of the single item trait empathy scale (SITES). J. Res. Pers. 73, 111-122. doi: 10.1016/j.jrp.2017.11.009

${ }^{*}$ Kose, S., Celikel, F. C., Kulacaoglu, F., Akin, E., Yalcin, M., and Ceylan, V. (2018). Reliability, validity, and factorial structure of the Turkish version of the Empathy Quotient (Turkish EQ). Psychiatry Clin. Psychopharmacol. 28, 300-305.

${ }^{*}$ Kosonogov, V. (2014). The psychometric properties of the Russian version of the empathy quotient. Psychol. Russia 7:96.

Lawrence, E. J., Shaw, P., Baker, D., Baron-Cohen, S., and David, A. S. (2004). Measuring empathy: reliability and validity of the Empathy Quotient. Psychol. Med. 34:911. doi: 10.1017/s0033291703001624

${ }^{*}$ Liang, Y. S., Yang, H. X., Ma, Y. T., Lui, S. S., Cheung, E. F., Wang, Y., et al. (2019). Validation and extension of the questionnaire of cognitive and affective empathy in the Chinese setting. PsyCh J. 8, 439-448. doi: 10.1002/pchj.281

${ }^{*}$ Lietz, C. A., Gerdes, K. E., Sun, F., Geiger, J. M., Wagaman, M. A., and Segal, E. A. (2011). The empathy assessment index (EAI): a confirmatory factor analysis of a multidimensional model of empathy. J. Soc. Soc. Work Res. 2, 104-124. doi: $10.5243 /$ jsswr.2011.6

${ }^{*}$ Lindeman, M., Koirikivi, I., and Lipsanen, J. (2018). Pictorial empathy test (PET): an easy-to-use method for assessing affective empathic reactions. Eur. J. Psychol. Assess. 34:421. doi: 10.1027/1015-5759/a000353

López-Pérez, B., Fernández, I., and Abad, F. J. (2008). TECA. Cuestionario de Empatía Cognitiva y Afectiva. Madrid: TEA.

${ }^{*}$ Lucas-Molina, B., Pérez-Albéniz, A., Ortuño-Sierra, J., and Fonseca-Pedrero, E. (2017). Dimensional structure and measurement invariance of the Interpersonal Reactivity Index (IRI) across gender. Psicothema 29, 590-595. doi: 10.7334/psicothema2017.19

Macchiavelli, A., Giffone, A., Ferrarello, F., and Paci, M. (2020). Reliability of the six-minute walk test in individuals with stroke: systematic review and meta-analysis. Neurol. Sci. 42, 81-87. doi: 10.1007/s10072-020-04829-0

Marsh, A. A. (2018). The neuroscience of empathy. Curr. Opin. Behav. Sci. 19, $110-115$.

${ }^{*}$ Mendez, R. V., Graham, R., Blocker, H., Harlow, J., and Campos, A. (2011). Construct validation of Mexican empathy scale yields a unique mexican factor. Acta Investig. Psicol. Psychol. Res. Rec. 1, 381-401.
${ }^{*}$ Miguel, F. K., Hashimoto, E. S., Gonçalves, E. R. D. S., Oliveira, G. T. D., and Wiltenburg, T. D. (2018). Validity studies of the online empathy questionnaire. Trends Psychol. 26, 2203-2216.

Moher, D., Liberati, A., Tetzlaff, J., and Altman, D. G. (2009). Preferred reporting items for systematic reviews and meta-analyses: the PRISMA statement. Ann. Intern. Med. 151, 264-269. doi: 10.7326/0003-4819-151-4-20090818000135

Morelli, S. A., Lieberman, M. D., and Zaki, J. (2015). The emerging study of positive empathy. Soc. Pers. Psychol. Compass 9, 57-68. doi: 10.1111/spc3.12157

Moya-Albiol, L., Herrero, N., and Bernal, M. C. (2010). Neural bases of empathy. Rev. Neurol. 50, 89-100.

Muncer, S. J., and Ling, J. (2006). Psychometric analysis of the empathy quotient (EQ) scale. Pers. Individ. Diff. 40, 1111-1119. doi: 10.1016/j.paid.2005. 09.020

${ }^{*}$ Murphy, B. A., Costello, T. H., Watts, A. L., Cheong, Y. F., Berg, J. M., and Lilienfeld, S. O. (2018). Strengths and weaknesses of two empathy measures: a comparison of the measurement precision, construct validity, and incremental validity of two multidimensional indices. Assessment 27, 246-260. doi: 10.1177/ 1073191118777636

${ }^{*}$ Myszkowski, N., Brunet-Gouet, E., Roux, P., Robieux, L., Malézieux, A., Boujut, E., et al. (2017). Is the questionnaire of cognitive and affective empathy measuring two or five dimensions? Evidence in a French sample. Psychiatry Res. 255, 292-296. doi: 10.1016/j.psychres.2017.05.047

*Oceja Fernández, L. V., López Pérez, B., Ambrona Benito, T., and Fernández, I. (2009). Measuring general dispositions to feeling empathy and distress. Psicothema 21, 171-176.

*Park, Y. E., Yoon, H. K., Kim, S. Y., Williamson, J., Wallraven, C., and Kang, J. (2019). A Preliminary Study for translation and validation of the korean version of the cognitive, affective, and somatic empathy scale in young adults. Psychiatry Investig. 16:671. doi: 10.30773/pi.2019.06.25

Pasquali, L. (2020). IRT - Item Theory Response: Theory, Procedures and Applications. Curitiba: Editora Appris.

Pentapati, K. C., Yeturu, S. K., and Siddiq, H. (2020). A reliability generalization meta-analysis of Child Oral Impacts On Daily Performances (C-OIDP) questionnaire. J. Oral Biol. Craniof. Res. 10, 776-781. doi: 10.1016/j.jobcr.2020. 10.017

*Preti, A., Vellante, M., Baron-Cohen, S., Zucca, G., Petretto, D. R., and Masala, C. (2011). The Empathy Quotient: a cross-cultural comparison of the Italian version. Cogn. Neuropsychiatry 16, 50-70. doi: 10.1080/13546801003790982

${ }^{*}$ Queirós, A., Fernandes, E., Reniers, R., Sampaio, A., Coutinho, J., and SearaCardoso, A. (2018). Psychometric properties of the questionnaire of cognitive and affective empathy in a Portuguese sample. PLoS One 13:e0197755. doi: 10.1371/journal.pone.0197755

Raine, A, and Chen, F. R. (2018). The cognitive, affective, and somatic empathy scales (CASES) for children. J. Clin. Child. Adolesc. Psychol. 47, 24-37.

Rasoal, C., Jungert, T., Hau, S., and Andersson, G. (2011). Development of a Swedish version of the scale of ethnocultural empathy. Psychology 2, 568-573. doi: 10.4236/psych.2011.26087

${ }^{*}$ Redondo, I., and Herrero-Fernández, D. (2018). Adaptation of the Empathy Quotient (EQ) in a Spanish sample. Terapia Psicol. 36, 81-89. doi: 10.1017/ SJP.2021.26

${ }^{\star}$ Reniers, R. L., Corcoran, R., Drake, R., Shryane, N. M., and Völlm, B. A. (2011). The QCAE: a questionnaire of cognitive and affective empathy. J. Pers. Assess. 93, 84-95. doi: 10.1080/00223891.2010.528484

${ }^{*}$ Rodrigues, J., Lopes, A., Giger, J. C., Gomes, A., Santos, J., and Gonçalves, G. (2011). Measure scales of empathizing/systemizing quotient: a validation test for the Portuguese population. Psicologia 25, 73-89.

*Sampaio, L. R., Guimarães, P. R. B., dos Santos, C. P., Formiga, N. S., and Menezes, I. G. (2011). Studies on the dimensionality of empathy: translation and adaptation of the Interpersonal Reactivity Index (IRI). Psico 42, $67-76$.

Scarpellini, G. R., Capellato, G., Rizzatti, F. G., da Silva, G. A., and Martinez, J. A. B. (2014). CARE scale of empaty: translation to the Portuguese spoken in Braziland initial validation results. Medicina 47, 51-58.

*Segal, E. A., Cimino, A. N., Gerdes, K. E., Harmon, J. K., and Wagaman, M. A. (2013). A confirmatory factor analysis of the interpersonal and social empathy index. J. Soc. Soc. Work Res. 4, 131-153. doi: 10.5243/jsswr.2013.9 
${ }^{*}$ Senese, V. P., De Nicola, A., Passaro, A., and Ruggiero, G. (2016). The factorial structure of a 15-item version of the Italian Empathy Quotient Scale. Eur. J. Psychol. Assess. 34, 344-351. doi: 10.1027/1015-5759/a000348

Siu, A. M., and Shek, D. T. (2005). Validation of the interpersonal reactivity index in a Chinese context. Res. Soc. Work Pract. 15, $118-126$.

*Spreng, R. N., McKinnon, M. C., Mar, R. A., and Levine, B. (2009). The toronto empathy questionnaire: scale development and initial validation of a factoranalytic solution to multiple empathy measures. J. Pers. Assess. 91, 62-71. doi: $10.1080 / 00223890802484381$

Surguladze, S., and Bergen-Cico, D. (2020). Empathy in a broader context: development, mechanisms, remediation. Front. Psychiatry 11:529. doi: 10.3389/ fpsyt.2020.00529

Thompson, B. (2004). Exploratory and Confirmatory Factor Analysis. Washington, DC: American Psychological Association.

*Totan, T., Dogan, T., and Sapmaz, F. (2012). The toronto empathy questionnaire: evaluation of psychometric properties among Turkish University Students. Eurasian J. Educ. Res. 46, 179-198.

${ }^{*}$ Vachon, D. D., and Lynam, D. R. (2016). Fixing the problem with empathy: development and validation of the affective and cognitive measure of empathy. Assessment 23, 135-149. doi: 10.1177/1073191114567941

Vitaglione, G. D., and Barnett, M. A. (2003). Assessing a new dimension of empathy: empathic anger as a predictor of helping and punishing desires. Motiv. Emot. 27, 301-325.

Vossen, H. G. M., Piotrowski, J. T., and Valkenburg, P. M. (2015). Development of the adolescent measure of empathy and sympathy (AMES). Pers. Individ. Diff. 4, 66-71. doi: 10.1016/j.paid.2014.09.040

${ }^{*}$ Wang, Y., Wen, Z., Fu, Y., and Zheng, L. (2017). Psychometric properties of a Chinese version of the measure of empathy and sympathy. Pers. Individ. Diff. $119,168-174$.

Warren, C. A. (2015). Scale of teacher empathy for African American males (S-TEAAM): measuring teacher conceptions and the application of empathy in multicultural classroom settings. J. Negro Educ. 84, 154-174.
*Wright, D. B., and Skagerberg, E. M. (2012). Measuring empathizing and systemizing with a large US sample. PLoS One 7:e31661. doi: 10.1371/journal. pone. 0031661

Yu, J., and Kirk, M. (2009). Evaluation of empathy measurement tools in nursing: systematic review. J. Adv. Nurs. 65, 1790-1806. doi: 10.1111/j.1365-2648.2009. 05071.x

${ }^{*}$ Yue, T., Wei, J., Huang, X., and Jiang, Y. (2016). Psychometric properties of the Chinese version of the Positive Empathy Scale among undergraduates. Soc. Behav. Pers. Int. J. 44, 131-138. doi: 10.2224/sbp.2016.44.1.131

*Zhang, Y., Xiang, J., Wen, J., Bian, W., Sun, L., and Bai, Z. (2018). Psychometric properties of the Chinese version of the empathy quotient among Chinese minority college students. Ann. Gen. Psychiatry 17:38. doi: 10.1186/s12991018-0209-Z

${ }^{\star}$ Zhao, Q., Neumann, D. L., Cao, X., Baron-Cohen, S., Sun, X., Cao, Y., et al. (2018). Validation of the empathy quotient in Mainland China. J. Pers. Assess. 100, 333-342. doi: 10.1080/00223891.2017.1324458

Conflict of Interest: The authors declare that the research was conducted in the absence of any commercial or financial relationships that could be construed as a potential conflict of interest.

Publisher's Note: All claims expressed in this article are solely those of the authors and do not necessarily represent those of their affiliated organizations, or those of the publisher, the editors and the reviewers. Any product that may be evaluated in this article, or claim that may be made by its manufacturer, is not guaranteed or endorsed by the publisher.

Copyright (c) 2021 Lima and Osório. This is an open-access article distributed under the terms of the Creative Commons Attribution License (CC BY). The use, distribution or reproduction in other forums is permitted, provided the original author(s) and the copyright owner(s) are credited and that the original publication in this journal is cited, in accordance with accepted academic practice. No use, distribution or reproduction is permitted which does not comply with these terms. 